Max-Planck-Institut für demografische Forschung

Max Planck Institute for Demographic Research

Konrad-Zuse-Strasse 1 - D-18057 Rostock · GERMANY

Tel +49 (0) 3812081 - 0; Fax +49 (0) 3812081 - 202;

http://www.demogr.mpg.de

MPIDR WORKING PAPER WP 2006-016

JUNE 2006

\title{
Scheidung in Ost- und Westdeutschland Der Einfluss der Frauenerwerbstätigkeit auf die Ehestabilität
}

Karin Böttcher (boettcher@ demogr.mpg.de)

This working paper has been approved for release by: Gerda Ruth Neyer (neyer@ demogr.mpg.de) Deputy Head of the Laboratory of Contemporary European Fertility and Family Dynamics.

(C) Copyright is held by the authors.

Working papers of the Max Planck Institute for Demographic Research receive only limited review. Views or opinions expressed in working papers are attributable to the authors and do not necessarily reflect those of the Institute. 


\section{SCHEIDUNG IN OST- UND WESTDEUTSCHLAND \\ Der Einfluss der Frauenerwerbstätigkeit auf die Ehestabilität}

\section{Zusammenfassung}

Dieser Beitrag vergleicht die Ehestabilität ost- und westdeutscher Frauen vor der Wiedervereinigung im Jahr 1990. Es wird untersucht, in welchem Ausmaß sich die Frauen beider Länder in Bezug auf das Risiko, eine erste Scheidung zu erfahren, unterschieden haben und auf welche Faktoren diese Unterschiede zurückzuführen sind. Im Mittelpunkt steht dabei der Einfluss der Frauenerwerbstätigkeit auf die Ehestabilität. Während der DDR, im Hinblick auf die Erwerbsbeteiligung der Frau, ein egalitäres Rollenverständnis zugrunde lag, unterstützten die gesellschaftlichen und institutionellen Rahmenbedingungen der BRD das traditionelle Modell eines männlichen Hauptverdieners. Die Gegensätzlichkeit dieser Rollenmuster ermöglicht es, zu untersuchen, ob hinsichtlich des Zusammenhangs von Frauenerwerbstätigkeit und Ehestabilität zwischen traditionell und egalitär ausgerichteten Gesellschaften Unterschiede bestehen. Die empirischen Analysen auf der Basis des deutschen „Family and Fertility Survey“ (1992) zeigen ein signifikant höheres Scheidungsrisiko ostdeutscher Frauen, welches in erster Linie auf die geringere Verbreitung religiöser Bindungen, den höheren Anteil von Frauen, die bereits eine Scheidung in der Elterngeneration erlebt haben und die höhere Frauenerwerbsbeteiligung in der DDR zurückzuführen ist. In beiden Ländern war die Erwerbstätigkeit der Frau mit einem höheren Scheidungsrisiko verbunden, der Effekt war in der BRD jedoch stärker als in der DDR. Diese Untersuchung liefert somit Beweise für einen schwächeren negativen Zusammenhang von Frauenerwerbstätigkeit und Ehestabilität in Gesellschaften, in denen egalitäre anstelle von traditionellen Rollenerwartungen dominieren.

\section{Stichworte}

Scheidung, Frauenerwerbstätigkeit, Ostdeutschland, Westdeutschland, FFS 


\section{Einleitung}

Ausgangspunkt vieler Argumentationen über den Zusammenhang von Frauenerwerbstätigkeit und Scheidungsrate ist die erstaunliche Parallelität in der Entwicklung der beiden Größen. In einer US-amerikanischen Untersuchung stellt Greenstein (1990: 659) fest:

„... we find that both indicators have been generally increasing since the late nineteenth century; that both indicators have increased sharply since the 1950s; and that the relative increases in the indicators have been approximately equal."

Auch für die Bundesrepublik Deutschland und für die Deutsche Demokratische Republik wurde dieser Zusammenhang bestätigt (Hill und Kopp 1994). Was ist jedoch die Ursache für das mit der Erwerbsbeteiligung der Frau ansteigende Scheidungsrisiko? Welche Mechanismen verbergen sich hinter diesem Zusammenhang? Ziel dieser Studie ist es, die Stabilität ost- und westdeutscher Ehen vor der Wiedervereinigung zu vergleichen. Es soll überprüft werden, in welchem Ausmaß sich Personen aus der DDR von Personen aus der BRD in Bezug auf das Risiko, eine erste Scheidung zu erfahren, unterschieden haben und auf welche Faktoren diese Unterschiede zurückzuführen sind. Im Mittelpunkt des Interesses steht dabei der Einfluss der Frauenerwerbstätigkeit auf die Ehestabilität.

Mit der DDR und der BRD entstanden nach dem zweiten Weltkrieg zwei Staaten, die zwar der gleiche kulturelle Hintergrund und eine gemeinsame Sprache verband, deren ideologische, wirtschaftliche und familienpolitische Entwicklung jedoch weitestgehend unterschiedlich verlief. In der DDR wurden bereits frühzeitig alle rechtlichen und institutionellen Rahmenbedingungen geschaffen, die eine Integration der Frau in das Erwerbsleben ermöglichten. Die Folge war eine lebenszeitlich fast kontinuierliche Vollzeiterwerbstätigkeit ostdeutscher Frauen. Im Gegensatz dazu unterstützte die bundesdeutsche Sozial- und Familienpolitik das traditionelle Modell eines männlichen Alleinverdieners. Finanzielle Anreize wie das Ehegattensplitting oder die Familienversicherung sowie fehlende Betreuungsangebote für Kinder förderten das „Hausfrauendasein“ westdeutscher Ehefrauen. Während die Erwerbstätigkeit beider Partner in ostdeutschen Ehen also Normalität war, stellte sie in Westdeutschland insbesondere für Ehepaare mit Kindern eher eine Ausnahme dar. Inwieweit beeinflussen jedoch die in einer Gesellschaft vorherrschenden Rollenerwartungen den Zusammenhang von Frauenerwerbstätigkeit und 
Ehestabilität? Gibt es im Hinblick auf den Einfluss, den die Erwerbstätigkeit der Frau auf die Ehestabilität ausübt, einen Unterschied zwischen egalitär (DDR) und traditionell (BRD) ausgerichteten Gesellschaften?

Zur Beantwortung dieser Frage wird in Abschnitt II zunächst ein Überblick über die gesellschaftlichen und institutionellen Rahmenbedingungen in Ost- und Westdeutschland gegeben. Daran anknüpfend wird für beide Länder die Entwicklung der Frauenerwerbstätigkeit und der Anstieg der Scheidungszahlen dargestellt. In Abschnitt III erfolgt eine systematische Zusammenfassung der Mechanismen, über welche die Erwerbstätigkeit der Frau einen Einfluss auf die Stabilität ehelicher Beziehungen ausübt. Darüber hinaus wird der bisherige Forschungsstand auf diesem Gebiet skizziert und es werden Hypothesen bezüglich des Zusammenhangs von Frauenerwerbstätigkeit und Ehestabilität in Ost- und Westdeutschland aufgestellt. Nach der Beschreibung des Datensatzes, der Variablen und der verwendeten Methode (Abschnitt IV), werden in Abschnitt V die Ergebnisse der empirischen Untersuchung vorgestellt. Abschnitt VI fasst die wichtigsten Ergebnisse dieser Untersuchung noch einmal zusammen.

\section{Gesellschaftliche und institutionelle Rahmenbedingungen in Ost- und Westdeutschland}

Sowohl in Ost- als auch in Westdeutschland wurde die Ehe als Grundlage familialen Zusammenlebens betrachtet, die durch familienpolitische Maßnahmen geschützt und gefördert werden sollte (Familiengesetzbuch der DDR 1989: §1 (1); Grundgesetz der BRD 2005: Art. 6 (1)). Während sich die westdeutsche Familienpolitik jedoch auf die Förderung des traditionellen Modells des männlichen Alleinverdieners konzentrierte und somit die Versorgungsfunktion der Ehe für die Frau unterstützte, standen in der ostdeutschen Familienpolitik die Erhaltung des Bevölkerungsbestandes und die Einbeziehung der Frauen in die Erwerbstätigkeit im Vordergrund (Bast und Ostner 1992). Die ökonomische Unabhängigkeit der Frau durch eigene Erwerbstätigkeit wurde in der DDR als Grundlage der Gleichberechtigung der Geschlechter angesehen (Koch und Knöbel 1988: 18). In der BRD hingegen wurde insbesondere während der Amtsperiode Franz-Josef Wuermelings (Bundesminister für Familienfragen von 1953 bis 1962) durch die Erwerbstätigkeit der Frau eine Gefährdung des familialen 
Zusammenhalts befürchtet (Münch 1990: 161f). Neben diesen ideellen Motiven hatte die unterschiedliche Bewertung und Förderung der Frauenerwerbstätigkeit in den zwei deutschen Staaten jedoch auch arbeitsmarktpolitische Gründe. Während die Erwerbstätigkeit der Frau in Ostdeutschland infolge des permanenten Arbeitskräftemangels eine dringende Notwendigkeit war (Koch und Knöbel 1988: 18), stellte sie in Westdeutschland, insbesondere zu Beginn der 50er Jahre und ab Mitte der 70er Jahre, aufgrund der angespannten Arbeitsmarktlage eher eine Belastung dar (Schäfgen 1998: 42-54).

Durch eine großzügige finanzielle Unterstützung von Familien mit Kindern und durch die Bereitstellung umfangreicher Kinderbetreuungsmöglichkeiten wurde Frauen in der DDR die Erfüllung ihres Kinderwunsches erleichtert und die Vereinbarkeit von Mutterschaft und Berufstätigkeit ermöglicht (Speigner 1989). Die BRD, insbesondere die ab 1982 regierende konservativ-liberale Koalition aus CDU und FDP, unterstützte dagegen das Konzept der Wahlfreiheit der Frau zwischen Beruf und Familie. Familienpolitische Maßnahmen, wie das Erziehungsgeld, das Ehegattensplitting und die Familienversicherung, aber auch die angespannte Arbeitsmarktlage und die unzureichende Versorgung mit Kinderbetreuungseinrichtungen, förderten den zeitweisen oder totalen Ausstieg der Frauen aus dem Erwerbsleben (Münch 1990: 172ff). Diesem familienpolitisch begünstigten Rückzug aus dem Erwerbsleben und dem daher oftmals nur diskontinuierlichen und von Teilzeit geprägten Erwerbsverlauf westdeutscher Frauen wurde in der Scheidungsgesetzgebung der BRD Rechnung getragen, indem Personen, die aufgrund von Kinderbetreuung, Alter oder Krankheit nicht arbeiten konnten bzw. keine angemessene Erwerbsarbeit fanden, Anspruch auf nachehelichen Unterhalt hatten. Westdeutsche Ehefrauen, die ihre Erwerbstätigkeit zugunsten von Kinderbetreuung und Hausarbeit aufgegeben hatten, sollten damit auch nach der Scheidung einen annehmbaren Lebensstandard aufrechterhalten können (Berghahn und Fritzsche 1991: 155ff). Die mit einer Scheidung verbundenen hohen Prozesskosten, die Aufteilung eines Einkommens auf zwei getrennte Haushalte sowie der Wegfall von Ehegattensplitting und Familienversicherung führten in Westdeutschland jedoch in vielen Fällen zu einer Verschlechterung der finanziellen Situation für alle Betroffenen (Wagner 1997: 159). Im Gegensatz zur BRD wurden nacheheliche Unterhaltszahlungen in der DDR nur in Ausnahmefällen und zeitlich begrenzt gewährt. Frauen sollten, unabhängig von ihrer familialen Situation, dazu in der Lage sein, ihren Lebensunterhalt durch eigene Erwerbsarbeit selbst zu verdienen. 
Auch die Dauer und die Kosten eines ostdeutschen Scheidungsverfahrens waren im Vergleich zu dem eines Westdeutschen gering (Berghahn und Fritzsche 1991: 141ff). Zusammenfassend lässt sich festhalten, dass es zwischen den zwei deutschen Staaten sowohl im Hinblick auf die Integration der Frauen in das Erwerbsleben als auch in Bezug auf die rechtliche Regulierung der Ehescheidung deutliche Unterschiede gegeben hat. Inwieweit haben diese unterschiedlichen Rahmenbedingungen jedoch das Verhalten der ost- und westdeutschen Bevölkerung beeinflusst?

\section{Frauenerwerbsbeteiligung in Ost- und Westdeutschland}

Infolge der stark abweichenden Bemühungen zur Integration der Frauen in das Erwerbsleben hat es zwischen Ost- und Westdeutschland im Hinblick auf die Entwicklung der Frauenerwerbsbeteiligung deutliche Unterschiede gegeben. In der DDR führten die intensiven, auf Frauen gerichteten arbeitsmarktpolitischen Kampagnen der 50er und 60er Jahre $\mathrm{zu}$ einem raschen Anstieg weiblicher Erwerbstätiger (siehe Abbildung 1). Zwischen 1950 und 1970 stieg die Erwerbsquote ${ }^{1}$ ostdeutscher Frauen um 29 Prozent, bis 1989 um weitere 8 Prozent. In Westdeutschland hingegen hat sich die Frauenerwerbsquote über den gesamten Zeitraum nur geringfügig erhöht und lag im Jahr 1989 mit 55,5 Prozent deutlich unter der ostdeutscher Frauen (89,0 Prozent). Darüber hinaus war der gestiegene Anteil weiblicher Erwerbstätiger in Westdeutschland in erster Linie die Folge eines Anstiegs geringfügig und teilzeitbeschäftigter Frauen (Deutsches Institut für Wirtschaftsforschung 1987).

\footnotetext{
${ }^{1}$ Unter einer Erwerbsquote versteht man den prozentualen Anteil der Erwerbspersonen (Erwerbstätige und Arbeitssuchende) an der Gesamtbevölkerung bzw. der Bevölkerung im arbeitsfähigen Alter. In diesem Fall handelt es sich um den Anteil der erwerbstätigen und Arbeit suchenden Frauen an allen Frauen im arbeitsfähigen Alter. Trotz eines unterschiedlichen gesetzlichen Renteneintrittsalters für Frauen wird, zum Zwecke einer besseren Vergleichbarkeit, für beide Länder als Bezugsgröße die weibliche Bevölkerung im Alter von 15 bis 65 Jahren verwendet.
} 
Abbildung 1: Entwicklung der Frauenerwerbsquote in Ost- und Westdeutschland (19501989)

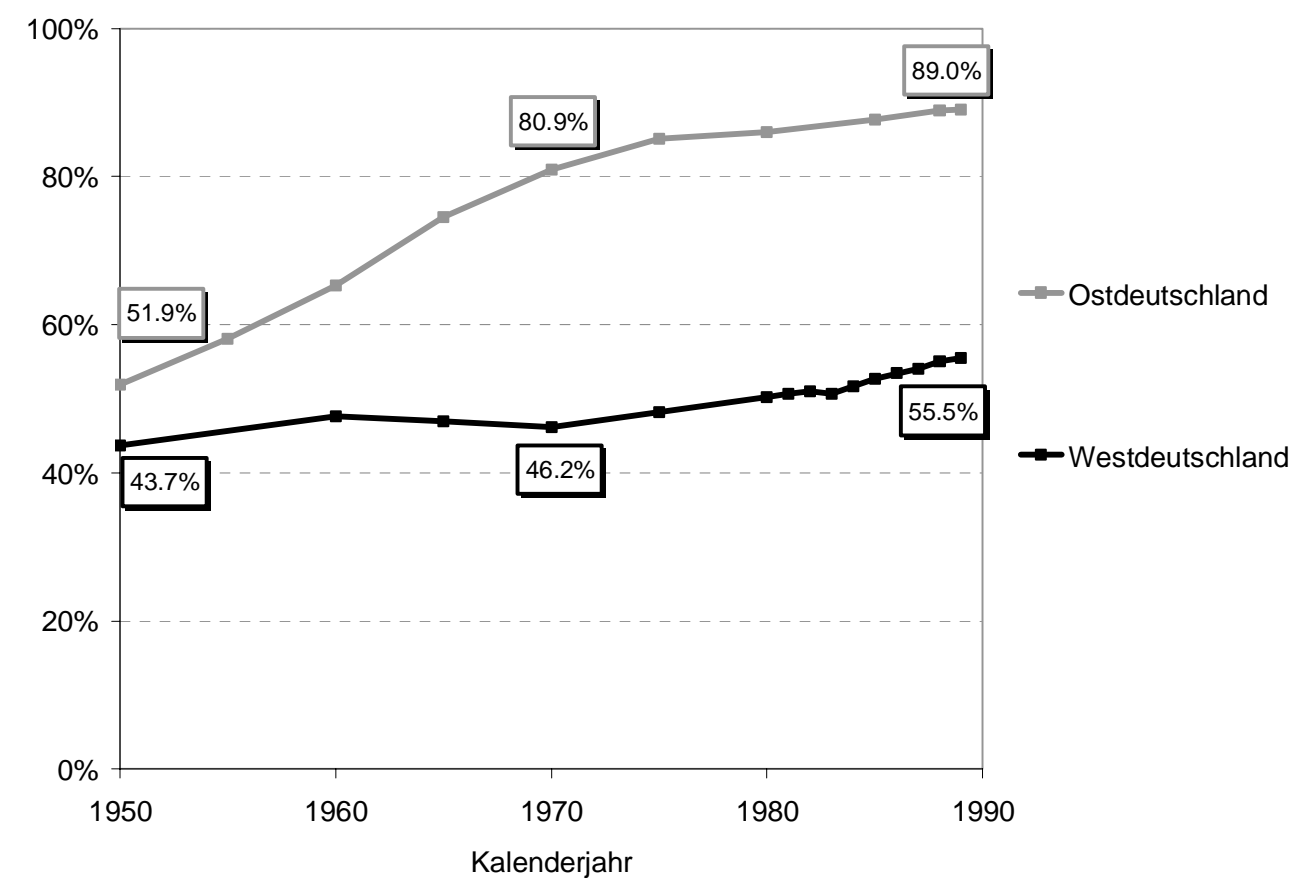

Quelle: Bothfeld et al. (2005)

Auch in der DDR gab es die Möglichkeit eine Teilzeitbeschäftigung auszuüben. Zu Beginn der 60er Jahre sollte damit nicht erwerbstätigen Frauen der Einstieg in die Erwerbsarbeit erleichtert werden. Nachdem die ostdeutschen Frauen Ende der 60er Jahre weitgehend in die Arbeitswelt integriert waren, versuchte man jedoch, der sich kontinuierlich ausbreitenden Teilzeitarbeit entgegenzuwirken. Sozialpolitische Maßnahmen, wie die Reduzierung der Arbeitszeit für vollzeitbeschäftigte Mütter mit mehreren Kindern, der Ausbau des Kinderbetreuungs- und Dienstleistungssystems sowie die zunehmende Propagierung der Vollzeitbeschäftigung als moralische Pflicht, sollten Teilzeit arbeitende Frauen zu einem Übergang in die Vollzeiterwerbstätigkeit bewegen (Trappe 1995: 144ff). Infolgedessen ging der Anteil teilzeitbeschäftigter ostdeutscher Frauen, der sich zwischen 1960 (15 Prozent) und 1970 (32 Prozent) mehr als verdoppelt hatte, ab Mitte der 70er Jahre langsam zurück und lag 1989 bei 27 Prozent (Winkler 1990: 83).

Während die Erwerbsbeteiligung der Frau in Ostdeutschland insbesondere in den jüngeren Geburtsjahrgangskohorten weitgehend unabhängig von der familialen Situation erfolgte (Trappe 1995: 117), wurde das Erwerbsverhalten westdeutscher Frauen in starkem Maße von dem Familienstand sowie der Anzahl und dem Alter der Kinder beeinflusst. Die höchsten Erwerbsquoten hatten in der BRD ledige und 
geschiedene Frauen (Lauterbach 1991). Die Geburt von Kindern führte in den meisten Fällen zu einem Rückzug westdeutscher Frauen aus dem Erwerbsleben. Dieses Verhalten war jedoch an den Familienstand der Ehe und die damit einhergehende Existenz eines männlichen Haupternährers gebunden (Deutsches Institut für Wirtschaftsforschung 1987).

\section{Ehescheidungen in Ost- und Westdeutschland}

Das Ausmaß der Ehescheidungen entwickelte sich in Ost- und Westdeutschland trotz der unterschiedlichen gesellschaftlichen und institutionellen Rahmenbedingungen weitgehend parallel (siehe Abbildung 2). Sowohl die allgemeine (Ehescheidungen je 10.000 Einwohner) als auch die spezifische Scheidungsziffer (Ehescheidungen je 10.000 Ehen) zeigt für beide Teile Deutschlands einen kontinuierlichen Anstieg der Scheidungshäufigkeit. Diese Entwicklung hat sich in Ostdeutschland jedoch über den gesamten Zeitraum auf einem deutlich höheren Niveau vollzogen. In der BRD ist die Scheidungshäufigkeit Ende der 70er, in der DDR Anfang der 90er Jahre abrupt abgesunken. Dabei ist der Einbruch der westdeutschen Scheidungsziffern auf die Reform des Scheidungsrechts im Jahr 1977 und die damit verbundene Änderung der verfahrensrechtlichen Bestimmungen zurückzuführen. ${ }^{2}$ In Ostdeutschland fand der drastische Rückgang der Scheidungszahlen im Zuge der Wiedervereinigung statt und wurde vermutlich in erster Linie durch die Übernahme des bundesdeutschen Eherechts verursacht, welches schnellen Scheidungen entgegenwirkt.

\footnotetext{
${ }^{2}$ Höhn (1980) weist darauf hin, dass nicht die seit 1977 erforderliche Trennungszeit von mindestens einem Jahr zu dem Einbruch der Scheidungszahlen geführt hat, sondern das seitdem angewandte Verbundverfahren und der damit einhergehende erhöhte Zeitaufwand. Während nach altem Recht im Rahmen des Scheidungsverfahrens nur die Scheidung und gegebenenfalls die Schuldfrage gerichtlich festgestellt wurde, muss nach neuem Recht im gleichen Verfahren neben der Scheidung auch das Sorge- und Umgangsrecht für minderjährige Kinder, der Kindes- und Ehegattenunterhalt sowie der Versorgungs- und Zugewinnausgleich entschieden werden.
} 
Abbildung 2: Entwicklung der allgemeinen und spezifischen Scheidungsziffer in Ost- und Westdeutschland (1950-1990)

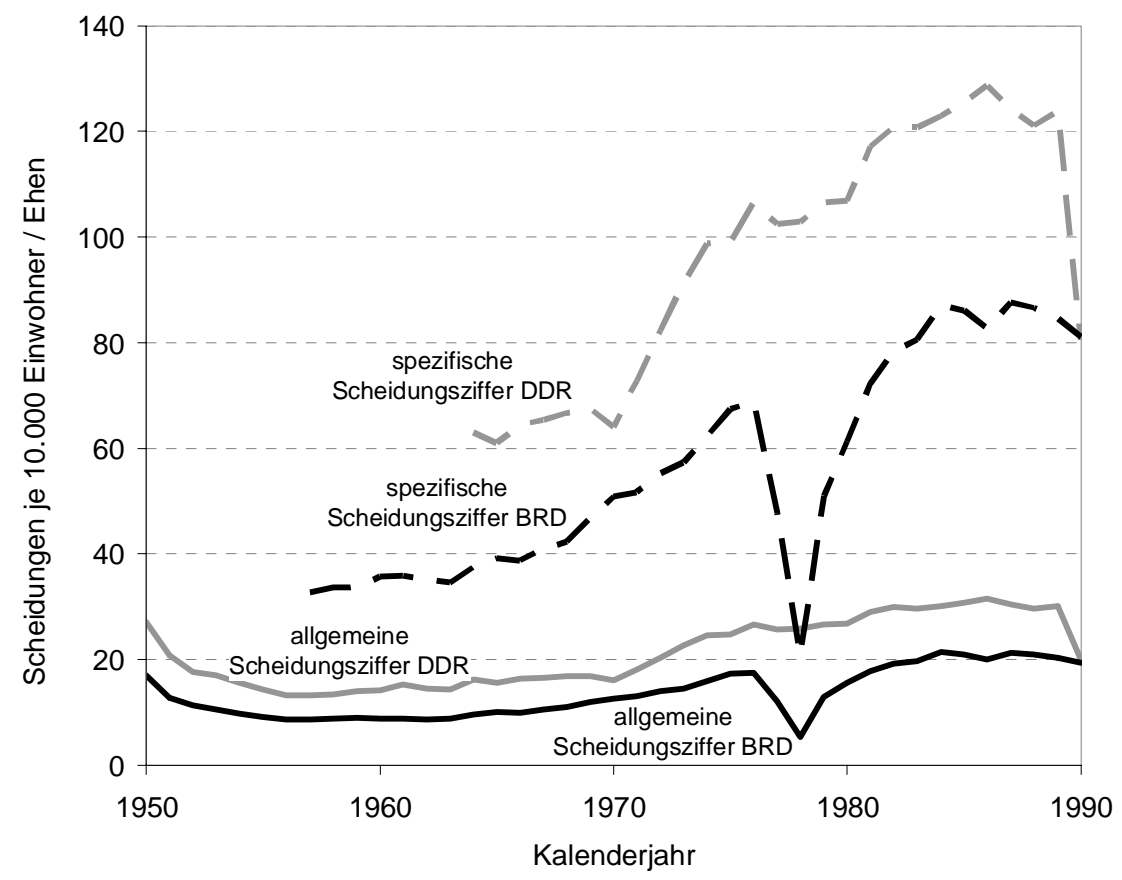

Quelle: Eurostat 2004; Hammes 1994: 978; Höhn 1977: 85; Staatliche Zentralverwaltung für Statistik 1964-1989; Statistisches Amt der DDR 1990b; Statistisches Bundesamt 1992; eigene Berechnungen

Querschnittsmaße, wie allgemeine oder spezifische Scheidungsziffern, liefern jedoch nur einen groben Überblick über die Entwicklung ehelicher Instabilität, da sie kohortenspezifische Verschiebungen vernachlässigen. Weit aussagekräftiger ist die Betrachtung der Scheidungshäufigkeit für einzelne Ehejahrgänge. Aus diesen Längsschnittdaten ist ersichtlich, dass es in Westdeutschland in der Abfolge der Ehejahrgänge einen kontinuierlichen Rückgang der Ehestabilität gegeben hat (siehe Abbildung 3). Während von den im Jahr 1950 geschlossenen westdeutschen Ehen nach 25 Jahren nur etwa 10 Prozent durch einer Scheidung aufgelöst waren, wies der westdeutsche Ehejahrgang 1965 nach 25 Jahren einen Scheidungsanteil von 19 Prozent auf, der des Jahrgangs 1975 lag bei nahezu 30 Prozent. Auch in den jüngeren westdeutschen Kohorten hat sich der Anstieg des Anteils geschiedener Ehen fortgesetzt. Für Ostdeutschland ist eine kohortenspezifische Betrachtung der Ehestabilität über einen so langen Zeitraum aufgrund der Datenlage nicht möglich. Vorhandene Daten zeigen jedoch auch für die DDR eine mit dem Eheschließungsjahr ansteigende Scheidungshäufigkeit (siehe Abbildung 4). Vergleicht man die kohortenspezifischen Daten der beiden Länder, so zeigt sich erneut eine geringere Stabilität ostdeutscher Ehen. Nach einer Ehedauer von 16 Jahren war in der DDR ein 
Viertel des Ehejahrgangs 1970 durch Scheidung getrennt. Damit lag der kumulierte Anteil geschiedener Ehen in Ostdeutschland 6,5 Prozent über dem westdeutscher Ehen des gleichen Jahrgangs und gleicher Ehedauer.

Abbildung 3: Kumulierter Anteil geschiedener Ehen für ausgewählte westdeutsche Ehejahrgänge

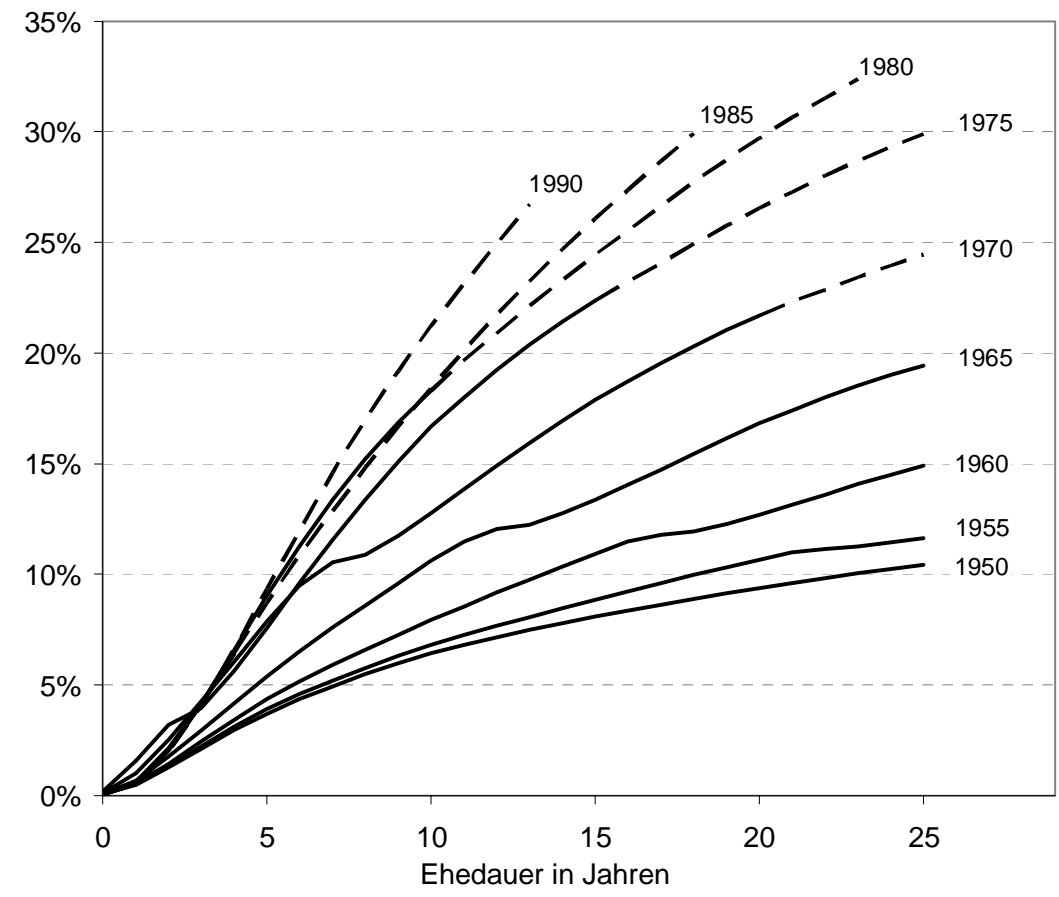

Quelle: Statistisches Bundesamt 2005

Anmerkungen: (1) Zur Verdeutlichung der langfristigen Entwicklung des kumulierten Anteils geschiedener Ehen an allen Ehen eines bestimmten Ehejahrgangs ist für Westdeutschland auch die Entwicklung nach 1990 in Form von gestrichelten Linien abgebildet. (2) Die niedrigen Scheidungszahlen Ende der 70er Jahre infolge der Reform des Ehescheidungsrechts sind verantwortlich dafür, dass der Anstieg des kumulierten Anteils geschiedener Ehen für die westdeutschen Ehejahrgänge 1955 bis 1975 zeitversetzt kurzfristig abnahm. 
Abbildung 4: Kumulierter Anteil geschiedener Ehen für ausgewählte ostdeutsche Ehejahrgänge

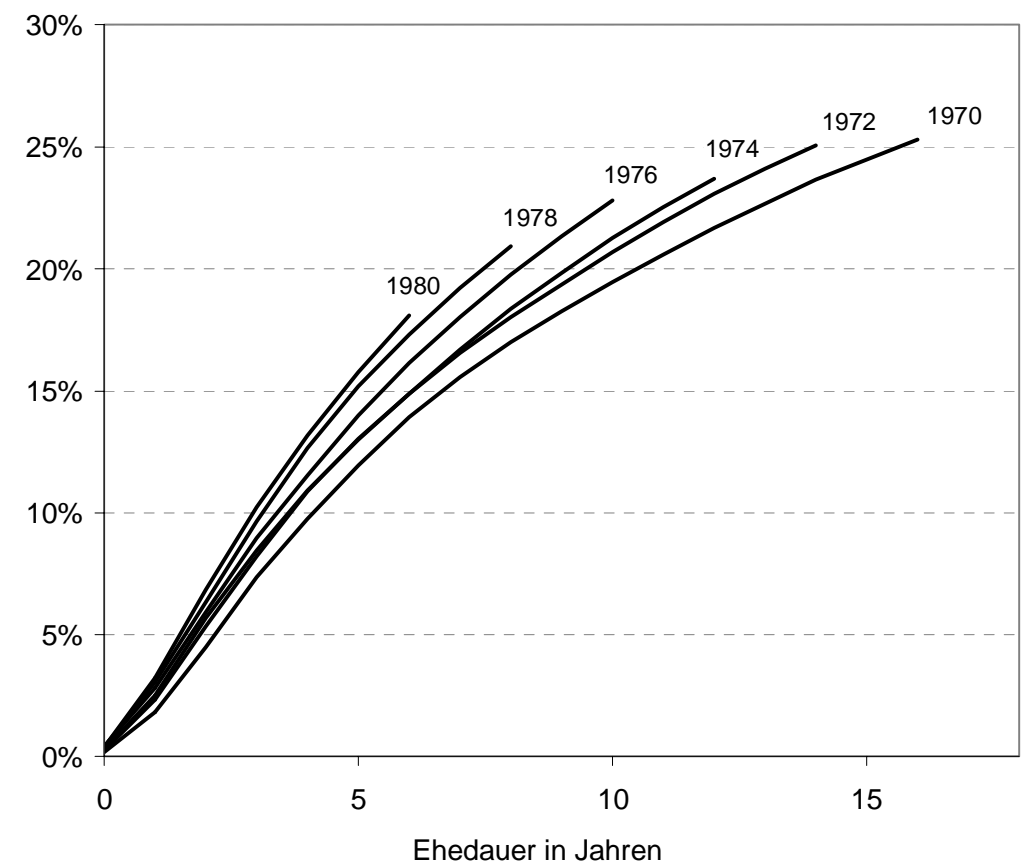

Quelle: Dobritz 1990, S. 70ff

Neben der Gemeinsamkeit eines kohortenspezifischen Anstiegs der Scheidungshäufigkeit ist demnach festzuhalten, dass es zwischen Ost- und Westdeutschland in Bezug auf das Ausmaß der Ehescheidungen erhebliche Unterschiede gab. So war das Scheidungsniveau in der DDR zu jedem Zeitpunkt höher als in der Bundesrepublik Deutschland. Inwieweit dies durch Abweichungen in den scheidungsrechtlichen Regelungen verursacht wurde, ist schwer zu beurteilen. Wagner (1997: 119) stellt jedoch fest, dass die ostdeutschen Scheidungsziffern schon vor der Gründung der DDR über denen in Westdeutschland lagen. Damit können Unterschiede im Scheidungsrecht nicht die einzige Ursache für das höhere Scheidungsniveau Ostdeutschlands gewesen sein.

\section{Der Zusammenhang zwischen Frauenerwerbstätigkeit und Ehestabilität}

Die Zunahme der Erwerbsbeteiligung von Frauen und der Anstieg des Scheidungsniveaus verliefen in vielen Staaten auffallend parallel (Hill und Kopp 1994). Vor diesem Hintergrund hat das wissenschaftliche Interesse an der Erforschung des Zusammenhangs von Frauenerwerbstätigkeit und Ehestabilität stark 
zugenommen. Vor allem im US-amerikanischen Raum wurden zahlreiche empirische Untersuchungen zu diesem Thema durchgeführt (vgl. z.B. D'Amico 1983; Booth et al. 1984; Greenstein 1990, 1995; Heckert et al. 1998; Sayer und Bianchi 2000; South 2001; Rogers 2004). Die Anzahl europäischer Studien ist dagegen vergleichsweise gering (vgl. für Deutschland Hartmann und Beck 1999; für Schweden Liu und Vikat 2004; für die Niederlande Kalmijn et al. 2004; für Spanien Houle 2005). Mit den möglichen Einflussmechanismen der Erwerbstätigkeit der Frau auf die eheliche Stabilität haben sich im deutschsprachigen Raum insbesondere Hill und Kopp (1994) sowie Hartmann und Beck (1999) auseinandergesetzt. Aufbauend auf diesen Arbeiten sollen im Folgenden die Zusammenhänge zwischen Frauenerwerbstätigkeit und Ehestabilität systematisiert und vervollständigt werden.

1. Mechanismen der Einflussnahme der Erwerbstätigkeit der Frau auf die Stabilität der ehelichen Beziehung

Sowohl in klassischen Arbeiten der Soziologie als auch in neueren Untersuchungen wird eine Vielzahl von Wirkungsmechanismen beschrieben, über die die Erwerbstätigkeit der Frau einen Einfluss auf die Stabilität der ehelichen Beziehung ausübt. Fasst man diese zusammen, so lassen sich insgesamt sieben unterschiedliche Effekte skizzieren.

Laut Parsons (1971: 266) entsteht durch die Erwerbstätigkeit der Frau zwischen den Ehepartnern ein Konkurrenzkampf hinsichtlich des gesellschaftlichen Status. Dieser Statuswettbewerb führt zwangsweise zu schweren Spannungen und damit zu einer erhöhten Instabilität der ehelichen Beziehung. Die geschlechtsspezifische Arbeitsteilung zwischen Mann und Frau ist für Parsons daher eine grundlegende Vorraussetzung für eheliche Stabilität.

Ein zweiter Wirkungsmechanismus kann aus der ökonomischen Theorie der Familie abgeleitet werden. Der Vorteil der Ehe gegenüber anderen Lebensformen besteht dieser Theorie zufolge unter anderem in der Möglichkeit einer arbeitsteiligen Organisation anfallender Aufgaben und der damit verbundene Effizienzsteigerung (Becker 1993: 30-53). Die Spezialisierung der Partner auf den Hausarbeits- oder Erwerbsarbeitssektor wird als eine Investition in ehespezifisches Kapital angesehen. Bleibt diese Investition aus, so sinkt über den Gewinn auch die Stabilität der 
ehelichen Beziehung. Auch Kinder spielen in diesem Zusammenhang eine wichtige Rolle (Becker 1977). Da eine Erwerbstätigkeit beider Partner aber oftmals mit dem Kinderwunsch konfligiert, wird dieser von berufstätigen Frauen hinausgezögert. Mit dem Verzicht auf Kinder, auch wenn er nur temporär geplant ist, fehlt jedoch eine weitere wichtige Investition in ehespezifisches Kapital (Hartmann und Beck 1999). Die Erwerbstätigkeit der Frau führt also in mehrfacher Hinsicht zu einer Verringerung ehespezifischer Investitionen und damit zu Instabilität.

Von der zeitlichen Inanspruchnahme der Frau durch die Erwerbstätigkeit geht eine weitere Gefahr für die Ehestabilität aus. Greenstein (1990, 1995) spricht in diesem Zusammenhang von einem destabilisierend wirkenden Abwesenheitseffekt weiblicher Erwerbstätigkeit. Zum einen steht erwerbstätigen Frauen weniger Zeit für Haushaltsund Familienarbeit zur Verfügung, wodurch sich die physische und psychische Belastung der Frau erhöht und gleichzeitig das Konfliktpotential infolge einer eventuell verminderten Qualität der Haushaltsarbeit steigt. Zum anderen besteht die Möglichkeit, dass unter erwerbstätigen Frauen Unzufriedenheit über die Aufteilung der häuslichen Tätigkeiten entsteht. So weist Greenstein darauf hin, dass Haushaltsarbeiten auch in Ehen, in denen die Frauen vollzeitbeschäftigt sind, in der Regel nicht von beiden Partnern in gleichem Ausmaß übernommen werden.

Während die physische und psychische Belastung der Frau steigt, nimmt für die Ehepartner die Möglichkeit, Zeit - vor allem Freizeit - gemeinsam zu verbringen ab (Hartmann und Beck 1999). Des Weiteren bleiben der Stress und die Probleme des Arbeitsalltages vermutlich nicht auf den Arbeitsplatz beschränkt, sondern beeinflussen auch das Ehe- und Familienleben (Hughes et al. 1992; Hill und Kopp 1994). Infolgedessen kommt es zu einer Beeinträchtigung der ehelichen Interaktion, die sich negativ auf die Stabilität der ehelichen Beziehung auswirkt. ${ }^{3}$

Ein weiterer die Ehestabilität reduzierender Wirkungsmechanismus ist die mit der Erwerbsbeteiligung der Frau verbundene Erhöhung der emotionalen Unabhängigkeit. So sind erwerbstätige Frauen aufgrund ihres Arbeitsumfeldes eher dazu in der Lage, sich einen vom gemeinsamen Netzwerk der Ehepartner unabhängigen Bekanntschaftskreis aufzubauen, wodurch auch die Chance, andere Partner kennen zu

\footnotetext{
${ }^{3}$ Es ist zwar davon auszugehen, dass auch die Erwerbstätigkeit von nur einem Partner von Zeit zu Zeit einen negativen Einfluss auf das Familienleben ausübt, Konflikte und Stresssituationen treten in diesem Fall jedoch seltener auf (da sie nur von einem Arbeitsplatz ausgehen können) und sind leichter zu bewältigen (da der nicht erwerbstätige Partner über genügend Zeit verfügt, um moralischen Beistand zu leisten und um dem Erwerbstätigen im Hinblick auf familiale Tätigkeiten den Rücken freizuhalten).
} 
lernen steigt. Darüber hinaus ist die identitätsstiftende Wirkung der Ehe für erwerbstätige Frauen vermutlich geringer, da sie sich nicht ausschließlich über ihre Rolle als Ehefrau bzw. Mutter, sondern auch über Berufserfahrungen definieren können (Spitze 1988; Hartmann und Beck 1999).

Ein besonderes Interesse gilt in Untersuchungen zum Thema Frauenerwerbstätigkeit und Ehestabilität dem Einfluss des mit der Erwerbstätigkeit erzielten Einkommens der Frau. Ross und Sawhill (1975: 35-66) sprechen in diesem Zusammenhang von zwei widersprüchlichen Effekten. Zum einen bewirkt das mit der Erwerbstätigkeit verbundene eigene Einkommen eine Verminderung der ökonomischen Abhängigkeit vom Ehemann. Insbesondere für Frauen, mit einem im Vergleich zum Ehemann relativ hohen Einkommen, nimmt der Nutzen der Ehe ab, während gleichzeitig die Möglichkeit, eine unglückliche Beziehung zu verlassen, steigt (Greenstein 1990; Sayer und Bianchi 2000). Das mit der Erwerbsarbeit verbundene Einkommen der Frau reduziert also über den so genannten Selbständigkeitseffekt die eheliche Stabilität. Zum anderen sind mit der Erwerbstätigkeit der Frau aber auch Aspekte verbunden, die sich positiv auf die Ehestabilität auswirken. So erhöht das Einkommen der Frau den finanziellen Spielraum und damit den Lebensstandard der Familie. Darüber hinaus begünstigt es die Akkumulation von ehespezifischem Kapital, soweit sich dieses auf materielle Güter wie zum Beispiel Wohneigentum bezieht. Über den Einkommenseffekt geht von der Erwerbstätigkeit der Frau also auch ein positiver, die Ehe stabilisierender Einfluss aus (Greenstein 1990; Kopp 1994). Es wird aber allgemein angenommen, dass der die Instabilität von Ehen fördernde Selbständigkeitseffekt jeglichen stabilisierend wirkenden Einkommenseffekt aufwiegt (Ross und Sawhill 1975: 54; Oppenheimer 1997).

Vor dem Hintergrund sich wandelnder Rollenbilder wird von mehreren Autoren die Gültigkeit einiger Effekte in Frage gestellt (vgl. z. B. Greenstein 1995; Oppenheimer 1997; Sayer und Bianchi 2000). Hill und Kopp (1994) weisen darauf hin, dass fast alle Effekte implizit oder explizit eine traditionelle Rollenteilung und ein traditionellen Orientierungen verpflichtetes Selbst- und Familienverständnis unterstellen. In egalitär ausgerichteten Partnerschaften hingegen, in denen die Erwerbstätigkeit beider Ehepartner den normativen Rollenerwartungen entspricht, sollten einige der negativen Effekte der Frauenerwerbstätigkeit kaum noch zu erwarten sein. So wird in egalitären Partnerschaften die Norm des männlichen 
Alleinverdieners durch den Grundsatz der gleichberechtigten Teilnahme am Erwerbsleben ersetzt. Damit stellt die Erwerbstätigkeit der Frau in diesen Partnerschaften keine Beeinträchtigung des männlichen Status dar, wodurch auch der negative Effekt eines Statuswettbewerbes nicht auftreten dürfte. Anzunehmen ist auch, dass der Ehemann bei egalitären Rollenerwartungen eine verminderte Qualität der Erledigung der häuslichen Tätigkeiten eher akzeptieren bzw. sich in größerem Ausmaß an der Hausarbeit beteiligen wird (Hartmann und Beck 1999). Von diesem Standpunkt aus dürfte der negative Einfluss des Abwesenheitseffektes in egalitären Partnerschaften also geringer sein. Andererseits werden Haushaltsaufgaben jedoch auch in Ehen, in denen beide Partner erwerbstätig sind, größtenteils von den Frauen erledigt. Die Mehrbelastung durch Erwerbs- und Hausarbeit bei fehlender bzw. ungenügender häuslicher Unterstützung durch den Ehemann wird von Frauen mit egalitären Rollenerwartungen wahrscheinlich eher als ungerecht empfunden werden, als von Frauen mit traditionellen Rollenerwartungen (Greenstein 1995). Vor dem Hintergrund dieser Überlegungen, ist in egalitären Partnerschaften eher ein noch stärkerer negativer Einfluss des Abwesenheitseffektes auf die Ehestabilität zu vermuten. Die ökonomische Unabhängigkeit der Frau wird von Personen mit egalitären Rollenvorstellungen als eine beabsichtigte Konsequenz der Erwerbstätigkeit beider Partner angesehen, da sie die Gleichberechtigung und Gleichbehandlung der Geschlechter fördert (Hill und Kopp 1994). Unter diesen Umständen geht vom Einkommen der Frau vermutlich auch kein die Stabilität der Ehe bedrohender Selbständigkeitseffekt, sondern nur ein stabilisierend wirkender Einkommenseffekt aus. Abbildung 5 fasst die Mechanismen der Einflussnahme der Frauenerwerbstätigkeit auf die Stabilität ehelicher Beziehungen sowohl für traditionelle als auch für egalitäre Partnerschaften zusammen. 
Abbildung 5: Mechanismen der Einflussnahme der Frauenerwerbstätigkeit auf die Ehestabilität

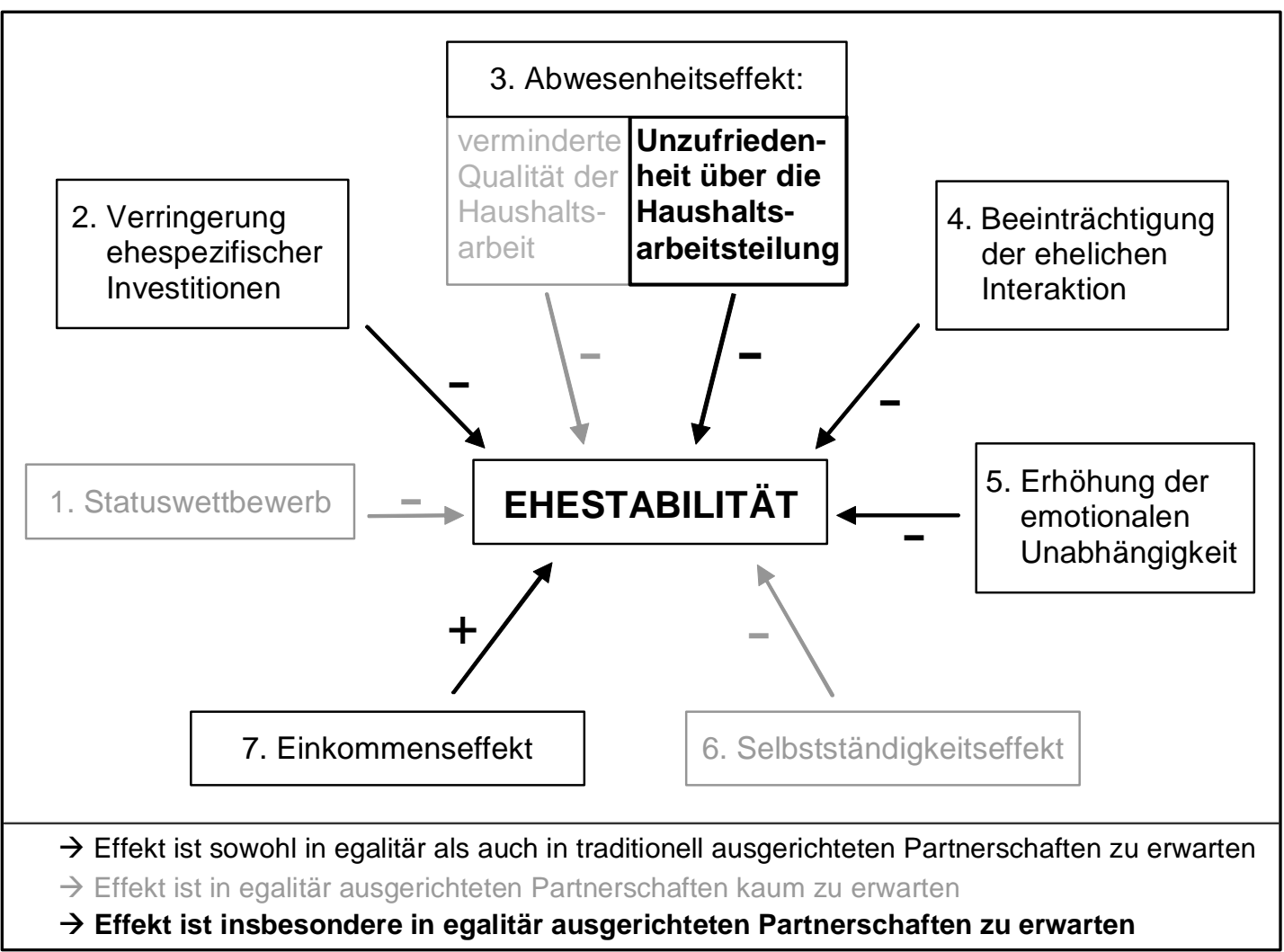

Es lässt sich also festhalten, dass die Erwerbstätigkeit der Frau die Stabilität ehelicher Beziehungen über eine Reihe unterschiedlicher Wirkungsmechanismen sowohl positiv als auch negativ beeinflusst. Dabei ist die Anzahl der negativen, destabilisierend wirkenden Effekte weiblicher Erwerbstätigkeit jedoch wesentlich größer, unabhängig davon, ob die Partnerschaft auf egalitären oder traditionellen Rollenvorstellungen basiert. Der destabilisierend wirkende Einfluss eines Statuswettbewerbes, einer verminderten Qualität der Haushaltsarbeit und eines Selbständigkeitseffektes ist in egalitären Partnerschaften kaum zu erwarten. Dagegen sollte die Unzufriedenheit der Frau über die häusliche Arbeitsteilung insbesondere in Partnerschaften mit egalitären Rollenbildern zu einer verstärkten Abnahme der ehelichen Stabilität führen. Insgesamt ist daher anzunehmen, dass der negative Effekt der Frauenerwerbstätigkeit auf die Ehestabilität abgeschwächt wird, wenn es sich um eine auf egalitären Rollenvorstellungen beruhende Partnerschaft handelt. 


\section{Ein Überblick über die bisherige Forschung}

Der Einfluss der Erwerbsbeteiligung der Frau auf die Ehestabilität wird in der Regel entweder über eine dichotome Variable mit den Ausprägungen „,erwerbstätig“ und „,nicht erwerbstätig“, über die Anzahl der Wochenarbeitsstunden oder über das mit der Erwerbstätigkeit verbundene absolute bzw. relative Einkommen der Frau analysiert. ${ }^{4}$ Viele US-amerikanische Studien zeigen einen negativen Zusammenhang zwischen dem Umfang der weiblichen Erwerbsbeteiligung und der ehelichen Stabilität (vgl. z.B. Mott und Moore 1979; Booth et al. 1984; South und Spitze 1986; Greenstein 1990, 1995). Greenstein (1995) ermittelte beispielsweise, dass Frauen mit einer Wochenarbeitszeit von 20 bis 35 Stunden im Durchschnitt ein 23 Prozent höheres Scheidungsrisiko haben als nicht erwerbstätige Frauen. Für Frauen mit einer Arbeitszeit von mehr als 40 Wochenstunden steigt das Scheidungsrisiko gegenüber Frauen, die 20 bis 35 Wochenstunden arbeiten, um 46 Prozent, gegenüber nicht erwerbstätigen Frauen um 80 Prozent. Kalmijn et al. (2004) untersuchten für die Niederlande den Einfluss von kulturellen und ökonomischen Faktoren auf das Scheidungsrisiko. Einen signifikant negativen Effekt der Erwerbsbeteiligung der Frau auf die Ehestabilität fanden die Autoren zwar für vollzeitbeschäftigte, nicht jedoch für teilzeitbeschäftigte Frauen. Sowohl Greenstein (1995) als auch Kalmijn et al. (2004) haben überprüft, inwieweit sich der Zusammenhang zwischen Frauenerwerbstätigkeit und Ehestabilität verändert, wenn die Existenz unterschiedlicher Rollenbilder berücksichtigt wird. Kalmijn et al. konnten einen negativen Einfluss der Frauenerwerbsbeteilung auf die Ehestabilität nur für Frauen mit traditionellen Rollenvorstellungen nachweisen und bestätigen damit die Vermutung, dass die destabilisierende Wirkung weiblicher Erwerbstätigkeit unter egalitär ausgerichteten Paaren schwächer ist. Greenstein kommt jedoch zu entgegen gesetzten Ergebnissen. In seiner Untersuchung weisen lediglich erwerbstätige Frauen mit egalitären Rollenvorstellungen ein signifikant höheres Scheidungsrisiko auf. Greenstein sieht darin eine Bestätigung dafür, dass die Unzufriedenheit der Frau über die häusliche Arbeitsteilung unter egalitären Rollenvorstellungen eher zu Instabilität führt als unter traditionellen Rollenvorstellungen.

\footnotetext{
${ }^{4}$ Unter dem relativen Einkommen der Frau wird in der Regel der Anteil verstanden, den die Frau zum Gesamteinkommen der Ehepartner beiträgt.
} 
Zur Untersuchung des Einflusses des (relativen) Einkommens der Frau auf die Stabilität ehelicher Beziehungen wurde in den USA bereits eine Vielzahl unterschiedlicher Datensätze und Analysemethoden verwendet. Die Ergebnisse sind allerdings widersprüchlich (Rogers 2004). Während einige Studien ein mit dem (relativen) Einkommen der Frau ansteigendes Scheidungsrisiko belegen (vgl. z.B. Spitze und South 1985; Hiedemann et al. 1998; Rogers 2004), konnte dieser Zusammenhang in anderen Studien nicht nachgewiesen werden (vgl. z.B. Greenstein 1990, 1995; South und Lloyd 1995). Sayer und Bianchi (2000) stellen zwar einen negativen Effekt des relativen Einkommens der Frau auf die Ehestabilität fest, dieser verliert jedoch seine Signifikanz sobald die Autorinnen eine Variable zur Berücksichtigung unterschiedlicher Rollenbilder in das Model einbeziehen. Einige US-amerikanische Untersuchungen deuten auf eine u-förmige Beziehung zwischen dem relativen Einkommen der Frau und dem Scheidungsrisiko hin, bei der das Risiko einer Ehescheidung am niedrigsten ist, wenn beide Partner annähernd gleich viel zum Gesamteinkommen der Ehe beitragen (vgl. z.B. Ono 1998). Andere Studien belegen hingegen, dass insbesondere diese Ehen von Instabilität bedroht sind (vgl. z.B. Heckert et al. 1998; Rogers 2004). Liu und Vikat (2004) haben für Schweden den Einfluss des relativen Einkommens der Frau auf die Ehestabilität untersucht. Ihren Ergebnissen zufolge steigt das Scheidungsrisiko linear mit dem Anteil, den die Frau zum Gesamteinkommen der Ehepartner beiträgt. Schwedische Frauen, die mehr als 80 Prozent des Gesamteinkommens verdienen, haben ein doppelt so hohes Scheidungsrisiko wie Frauen mit einem Einkommensbeitrag von unter 20 Prozent. Überraschenderweise kann also ein die Ehe destabilisierender Selbständigkeitseffekt weiblicher Erwerbstätigkeit auch für Schweden, ein Land in dem egalitäre Rollenbilder dominieren, nachgewiesen werden. Neben dem destabilisierend wirkenden Selbständigkeitseffekt belegen Liu und Vikat jedoch auch einen die Ehe stabilisierenden Einkommenseffekt weiblicher Erwerbstätigkeit. Demnach sind in Schweden insbesondere Ehen mit einem niedrigen Gesamteinkommen - im Vergleich zu Ehen mit einem mittleren oder hohen Einkommen - von Instabilität bedroht. Kalmijn et al. (2005) überprüften für die Niederlande den Einfluss des relativen Einkommens der Frau auf die Stabilität ehelicher und nichtehelicher Beziehungen. Sie stellen fest, dass der Zusammenhang zwischen dem relativen Einkommen der Frau und dem Scheidungs- bzw. Trennungsrisiko in Abhängigkeit vom Institutionalisierungsgrad der Beziehung variiert. Während die Stabilität ehelicher 
Beziehungen bei steigendem relativen Einkommen der Frau kontinuierlich sinkt, insbesondere bei einem Einkommensanteil der Frau von über 50 Prozent, sind nichteheliche Partnerschaften eher durch einen u-förmigen Verlauf des Trennungsrisikos gekennzeichnet. Die Autoren schlussfolgern daraus: „Specialization is beneficial to marriage, but equality is beneficial to cohabiting relationships“ (Kalmijn et al. 2005: 21).

Für Deutschland wurde der Einfluss weiblicher Erwerbstätigkeit auf die Stabilität ehelicher Beziehungen bisher lediglich in den Arbeiten von Ott (1992), Wagner (1997) sowie Hartmann und Beck (1999) untersucht. Während sowohl Ott als auch Hartmann und Beck für Westdeutschland einen negativen Effekt der Frauenerwerbstätigkeit auf die Ehestabilität feststellen, belegen die Ergebnisse Wagners einen Zusammenhang dieser Art nur für Ehen, die zwischen 1948 und 1974 geschlossen wurden. Nach 1975 geschlossene westdeutsche Ehen sind laut Wagner hingegen signifikant stabiler, wenn die Frau erwerbstätig ist. In Ostdeutschland hatten Hartmann und Beck zufolge sowohl vollzeit- als auch teilzeitbeschäftigte Frauen ein niedrigeres Scheidungsrisiko als nicht erwerbstätige Frauen (wobei diese Ergebnisse jedoch nicht signifikant sind). Wagner ermittelt für Ostdeutschland hingegen einen durchgängig negativen Einfluss der Erwerbstätigkeit der Frau auf die Ehestabilität. Signifikant ist dieser Zusammenhang aber nur für zwischen 1948 und 1974 geschlossene Ehen. Sowohl die Anzahl als auch die Aussagekraft existierender westund vor allem ostdeutscher Studien zum Zusammenhang von Frauenerwerbstätigkeit und Ehestabilität ist begrenzt. Darüber hinaus existieren für die zwei deutschen Staaten derzeit nahezu keine vergleichenden Analysen. Aus diesem Grund ist es schwierig, Aussagen darüber zu treffen, in welchem Ausmaß beispielsweise die höhere Frauenerwerbsbeteiligung in Ostdeutschland eine Ursache für die niedrigere Stabilität ostdeutscher Ehen war. Ebenso schwer zu beurteilen ist, inwieweit es zwischen Ost- und Westdeutschland hinsichtlich des Einflusses der Erwerbstätigkeit der Frau auf das Scheidungsrisiko Unterschiede gegeben hat. 
3. Theoretische Überlegungen zum Zusammenhang von Frauenerwerbstätigkeit und Ehestabilität in Ost- und Westdeutschland

Beim Vergleich der Ehestabilität verschiedener Länder müssen zwei Aspekte berücksichtigt werden. Einerseits können Abweichungen im Scheidungsniveau die Folge einer länderspezifischen Sozialstruktur sein, aufgrund derer der Anteil von Bevölkerungsgruppen mit hohem Scheidungsrisiko in den Ländern unterschiedlich hoch ist (Kompositionseffekte). Andererseits ist es jedoch auch möglich, dass die postulierten Zusammenhänge - also die Einflüsse bestimmter Faktoren - in den untersuchten Ländern unterschiedlich stark sind (Verhaltenseffekte). Beide Aspekte sollen im Rahmen der empirischen Untersuchung beachtet werden.

In Abschnitt II wurde gezeigt, dass es zwischen Ost- und Westdeutschland im Hinblick auf die gesellschaftlichen und institutionellen Rahmenbedingungen deutliche Unterschiede gegeben hat. Während der DDR, zumindest im Hinblick auf die Erwerbsbeteiligung der Frau, ein egalitäres Rollenverständnis zugrunde lag, hielt die politische Führung der BRD, insbesondere die konservativ-liberale Regierung der 80er Jahre, an der traditionellen Rollenverteilung fest. Zwar lässt sich das staatlich gewünschte Rollenverhalten nicht mit den individuellen Rollenerwartungen der Ehepartner gleichsetzen, anzunehmen ist jedoch, dass egalitäre Rollenvorstellungen unter ostdeutschen Ehepaaren stärker verbreitet waren als unter westdeutschen Ehepaaren. Demzufolge sollte es zwischen den beiden deutschen Staaten hinsichtlich des Einflusses der Frauenerwerbstätigkeit auf die Ehestabilität Unterschiede gegeben haben. So ist von der Erwerbstätigkeit der Frau zwar vermutlich in beiden deutschen Staaten eine höhere emotionale Unabhängigkeit der Frau und, infolge der Doppelbelastung durch Haushalt und Beruf, eine Beeinträchtigung der ehelichen Interaktion ausgegangen, ein Konkurrenzkampf der Ehepartner um die Ernährerrolle (Statuswettbewerb) und Streitigkeiten im Falle einer verminderten Qualität der Haushaltsarbeit sind jedoch wahrscheinlich eher in Westdeutschland aufgetreten. Dagegen haben diese Effekte die Stabilität ostdeutscher Ehen aufgrund der egalitären Rollenvorstellungen vermutlich kaum beeinflusst. Darüber hinaus ist anzunehmen, dass die Erwerbstätigkeit der Frau in der BRD stärker als in der DDR zu einer Verringerung ehespezifischer Investitionen geführt hat. Während westdeutsche erwerbstätige Frauen ihren Kinderwunsch infolge der Unvereinbarkeit von Beruf und Familie möglicherweise aufgeschoben oder reduziert haben, trifft dies auf ostdeutsche 
Frauen nicht zu. Aufgrund der intensiv geförderten Vereinbarkeit von Erwerbs- und Familientätigkeit hatte die Erwerbsbeteiligung der Frau in Ostdeutschland vermutlich keine negativen Auswirkungen auf die Kinderzahl. Auch einen das Scheidungsrisiko erhöhenden Selbständigkeitseffekt hat es vermutlich nur in Westdeutschland gegeben, da lediglich dort die Erwerbstätigkeit der Frau und die damit verbundene ökonomische Unabhängigkeit eine von der Normalität abweichende Situation darstellte. Eine ungleiche Verteilung der Haushaltsarbeit wird dagegen insbesondere unter egalitären Rollenerwartungen als ungerecht empfunden. Demzufolge sollte dieser Effekt vor allem für ostdeutsche erwerbstätige Frauen das Scheidungsrisiko erhöht haben. Unter den traditionellen westdeutschen Rollenvorstellungen wurde eine geringe Beteiligung der Männer an der Hausarbeit wahrscheinlich eher als normal empfunden - auch wenn beide Partner erwerbstätig waren - und hatte damit keinen destabilisierenden Einfluss. Für beide deutschen Staaten ist hingegen anzunehmen, dass sich das mit der Erwerbstätigkeit der Frau verbundene zusätzliche Einkommen positiv auf die Ehestabilität ausgewirkt hat. Während jedoch ein zweites Einkommen für ostdeutsche Ehepaare aufgrund der niedrigen Lohnstruktur von enormer Bedeutung war, wurde der finanzielle Zugewinn für westdeutsche Ehen durch den Wegfall familienpolitischer Leistungen (Ehegattensplitting etc.) stark reduziert. Daher war der positive, die Ehe stabilisierende Einkommenseffekt weiblicher Erwerbstätigkeit in der DDR vermutlich stärker als in der BRD.

Zusammenfassend lässt sich festhalten, dass von der Erwerbstätigkeit der Frau in Ostdeutschland vermutlich eine geringere Anzahl destabilisierend wirkender Effekte und ein stärkerer die Ehe stabilisierender Einkommenseffekt ausging. In dieser Untersuchung wird daher angenommen, dass der negative Zusammenhang zwischen Frauenerwerbstätigkeit und Ehestabilität in Ostdeutschland schwächer war als in Westdeutschland. Nach wie vor sollten jedoch erwerbstätige Frauen in beiden Teilen Deutschlands einem höheren Scheidungsrisiko ausgesetzt gewesen sein als nicht erwerbstätige Frauen. Neben dem unterschiedlich starken negativen Effekt der Frauenerwerbsbeteiligung auf die Ehestabilität (Verhaltenseffekt) existierten zwischen Ost- und Westdeutschland vermutlich auch Abweichungen hinsichtlich der das Scheidungsrisiko beeinflussenden sozialstrukturellen Zusammensetzung der Bevölkerung. Unter Berücksichtigung dieser Kompositionseffekte sollten sich vorhandene Unterschiede im ost- und westdeutschen Scheidungsniveau reduzieren. 
Die höhere Erwerbsbeteiligung ostdeutscher Frauen spielt in diesem Zusammenhang vermutlich eine entscheidende Rolle.

\section{Datensatz und Methode}

\section{Datensatz}

Für die empirischen Analysen werden Daten des deutschen „Family and Fertility Survey“ (FFS) verwendet. Beim FFS handelt es sich um eine international vergleichbare, retrospektiv angelegte Befragung $\mathrm{zu}$ Partnerschafts- und Erwerbsbiografien, Lebensstilen, aber auch zum Thema Kinderwunsch und Familienplanung (Pohl 1995: 1). Das Projekt wurde 1988 initiiert und seitdem in 24 Mitgliedsländern der Wirtschaftskommission der Vereinten Nationen für Europa durchgeführt (Bundesinstitut für Bevölkerungsforschung 2003). Auf der Basis der Empfehlungen der internationalen FFS-Arbeitsgruppe wurde der deutsche FFS vom Bundesinstitut für Bevölkerungsforschung vorbereitet. Die Datenerhebung erfolgte in Zusammenarbeit mit dem Bielefelder EMNID-Institut im Sommer 1992. Aufgrund der Abweichungen des deutschen Fragebogens vom FFS-Standardfragebogen waren zur Gewährleistung der internationalen Vergleichbarkeit umfangreiche Variablen- und Valenzenrecodierungen notwendig. Der damit verbundene Informationsverlust wurde ausgeglichen, indem zusätzlich zum reduzierten, standardisierten Datensatz ein durch die Originalinformationen ergänzter nationaler Datensatz erstellt wurde. Insgesamt wurden im deutschen FFS 10.012 in Privathaushalten lebende Personen deutscher Nationalität befragt, die zwischen 1952 und 1972 geboren und somit zum Zeitpunkt des Interviews zwischen 20 und 39 Jahre alt waren. Jeweils die Hälfte der Interviews wurde im früheren Bundesgebiet und in der ehemaligen DDR durchgeführt, wobei jeweils 30 Prozent Frauen und 20 Prozent Männer befragt wurden (Pohl 1995: 6ff).

Im Rahmen dieser Studie wird mit den Daten des nationalen FFS gearbeitet. Da im Falle eines männlichen Befragten nur geringe Informationen über die Erwerbstätigkeit der Frau existieren und sich diese auch nur auf die aktuelle Partnerin beziehen, sind in der verwendeten Stichprobe nur weibliche Befragte enthalten. Darüber hinaus wird in dieser Untersuchung nur die erste Ehe der befragten Frauen berücksichtigt. Der Familienstand des Mannes zum Zeitpunkt der Eheschließung geht in die empirischen 
Analysen als Kontrollvariable ein. Von den 5.996 befragten Frauen im deutschen FFS waren 1.990 Frauen in Ost- und 1.583 Frauen in Westdeutschland mindestens einmal verheiratet. Alle Fälle, in denen die erste Eheschließung erst nach Oktober 1990 erfolgte (131 Fälle), in denen ein Erstheiratsalter von weniger als 16 Jahren berichtet wurde (25 Fälle) und für die keine bzw. widersprüchliche Angaben zum Heirats- oder Trennungsjahr vorliegen (264 Fälle) werden von der empirischen Analyse ausgeschlossen. Der bereinigte Datensatz enthält 1.821 ost- und 1.332 westdeutsche Frauen, die bis Oktober 1990 zumindest einmal geheiratet haben. Von diesen Frauen erlebten in Ostdeutschland 326 und in Westdeutschland 206 die Auflösung ihrer ersten Ehe.

\section{Variablen}

\section{Abhängige Variable}

Aufgrund von Datensatzbeschränkungen wird in dieser Untersuchung ehelicher Instabilität sowohl Trennung als auch Scheidung analysiert. Der Grund für diese Vorgehensweise ist, dass im deutschen FFS keine Informationen über das genaue Scheidungsdatum enthalten sind. Abgefragt wurden nur der Zeitpunkt, an dem das Zusammenleben der Partner endete und die Ursache dafür (Trennung, Scheidung, Tod des Partners, unfreiwilliges Auseinanderziehen). Damit lässt sich zwar unterscheiden, ob für eine befragte Person eine Trennung oder Scheidung vorliegt, es wird jedoch keine Auskunft darüber gegeben, ob sich Paare deren Zusammenleben aufgrund einer Trennung endete, später scheiden ließen. Eine Beschränkung der Analysen auf Scheidungen würde in der westdeutschen Stichprobe $\mathrm{zu}$ einer überproportional starken Abnahme der Ereignisse führen, da der Anteil von Trennungen an der Gesamtzahl der aufgrund von Trennung oder Scheidung vom ersten Ehemann getrennt lebenden Frauen in der westdeutschen Stichprobe (20,9 Prozent) viermal so hoch ist wie in der ostdeutschen Stichprobe (5,2 Prozent). ${ }^{5}$ Zwar besteht die Möglichkeit, dass Paare, die sich getrennt haben, später wieder zusammenfinden, dies

\footnotetext{
${ }^{5}$ Ursache hierfür ist vermutlich, dass es in Westdeutschland aufgrund der besseren Verfügbarkeit von Wohnraum eher möglich war, bereits vor der Scheidung getrennt zu leben. Darüber hinaus erforderte das westdeutsche Scheidungsrecht sogar die Einhaltung von Trennungszeiten. In Ostdeutschland konnten Eheleute, die sich trennen wollten, dagegen oftmals erst mit einem gültigen Scheidungsurteil getrennte Wohnungen beantragen (Berghahn und Fritzsche 1991: 139ff).
} 
trifft im deutschen FFS jedoch lediglich auf eine Befragte $\mathrm{zu}$ und kann somit vernachlässigt werden. In dieser Untersuchung wird daher die Auflösung von Ehen untersucht, unabhängig davon, ob die Ehepartner zum Zeitpunkt des Auseinanderziehens nur getrennt oder bereits geschieden waren. ${ }^{6}$ In beiden Fällen wird das Ende des gemeinsamen Zusammenlebens als Ende der Ehe behandelt. Die Begriffe Scheidung und Scheidungsrisiko werden im Folgenden unabhängig davon verwendet, ob das Zusammenleben der Partner durch Scheidung oder Trennung endete.

\section{Unabhängige Variablen}

Im Mittelpunkt der nachfolgenden empirischen Untersuchungen steht der Einfluss der Frauenerwerbstätigkeit auf die Stabilität ehelicher Beziehungen. Da der FFS keine Informationen über das Einkommen der befragten Person im Zeitverlauf enthält, kann der Zusammenhang zwischen weiblicher Erwerbstätigkeit und Ehestabilität nur über den Umfang der Erwerbsbeteiligung der Frau analysiert werden. Die zeitabhängig konstruierte Erwerbsbeteiligungsvariable ist dabei in drei Kategorien unterteilt: a) nicht erwerbstätig, b) Teilzeit erwerbstätig und c) Vollzeit erwerbstätig. Befragte werden als „Teilzeit erwerbstätig“ eingestuft, wenn sie eine durchschnittliche Wochenarbeitszeit von weniger als 35 Stunden angaben. Als „,nicht erwerbstätig“ gelten Frauen, die keiner beruflichen Tätigkeit nachgingen bzw. Frauen, die eine Erwerbsunterbrechung von mehr als sechs Monaten berichteten. In Abbildung 6 ist für beide Länder die Verteilung der Risikozeit auf die einzelnen Ausprägungen der Erwerbsbeteiligungsvariable dargestellt. Die Risikozeit von Frauen mit fehlenden Angaben zum Anfang, zum Ende oder zum Umfang der Erwerbstätigkeit (Kategorie „keine Angabe“) wird dabei nicht berücksichtigt.

\footnotetext{
${ }^{6}$ Brüderl und Engelhardt (1997) haben empirisch untersucht, welche Konsequenzen ein Festmachen der Eheauflösung an den Ereignissen Trennung bzw. Scheidung hat. Sie zeigen, dass die Ergebnisse weitestgehend invariant gegenüber der gewählten Auflösungsdefinition sind und sich Unterschiede nur ergeben, wenn die Schätzungen auf sehr kleinen Stichproben basieren.
} 
Abbildung 6: Prozentuale Verteilung der Risikozeit auf die einzelnen Ausprägungen der Erwerbsbeteiligungsvariable (ohne die Kategorie „keine Angabe“)

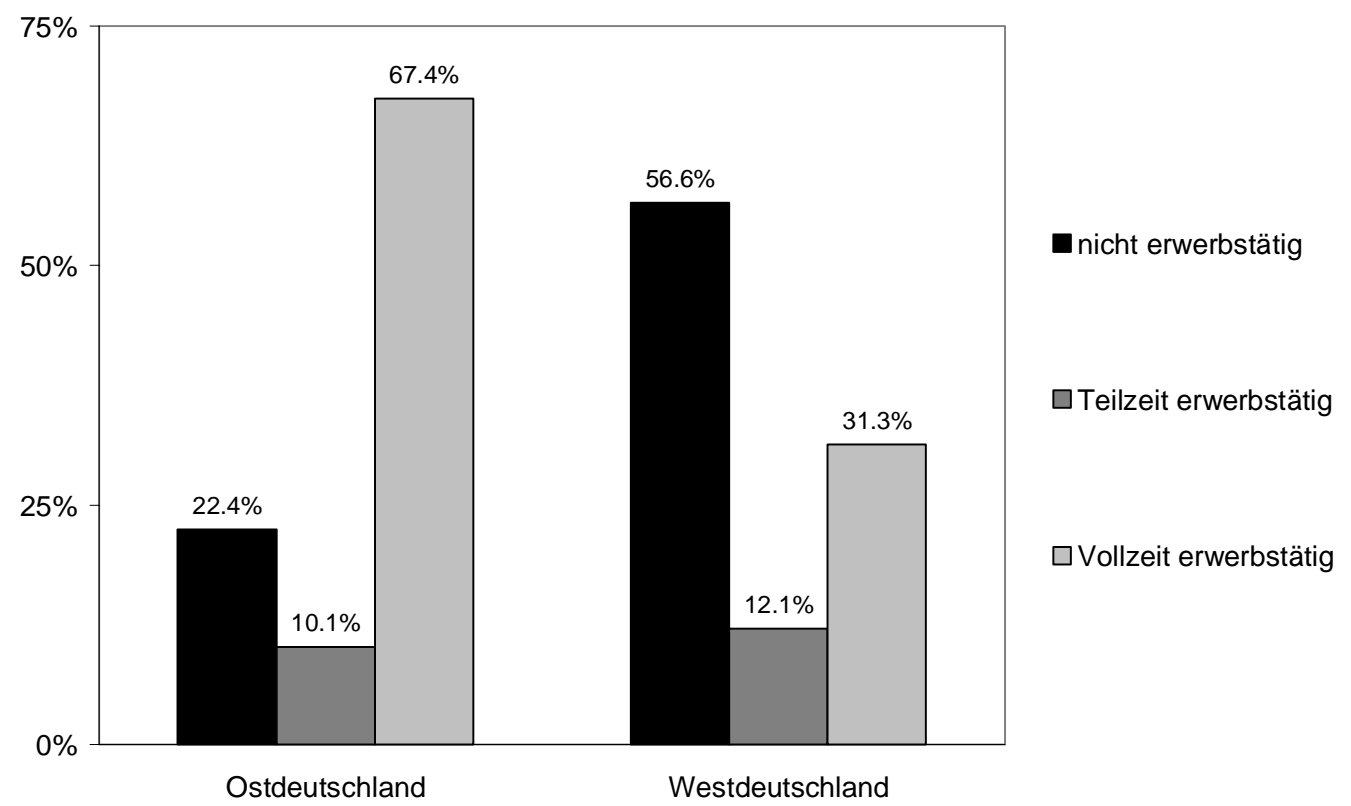

Quelle: nationaler FFS für Ost- und Westdeutschland, eigene Berechnungen

Von den ostdeutschen verheirateten Frauen wurden 22,4 Prozent der Risikozeit als „nicht erwerbstätig“ und 67,4 Prozent als „Vollzeit erwerbstätig“ durchlebt. Westdeutsche Ehefrauen verbrachten dagegen weniger als ein Drittel der Risikozeit als „Vollzeit erwerbstätig“ und knapp 57 Prozent als ,nicht erwerbstätig“. Im Hinblick auf den Anteil der Risikozeit, die verheiratete Frauen als „Teilzeit erwerbstätig“ durchlebten, gibt es zwischen den beiden Stichproben nur geringe Unterschiede.

Um zu überprüfen, ob es sich bei dem Einfluss der Frauenerwerbsbeteiligung auf die Ehestabilität um eine eigenständigen Effekt handelt und um herauszufinden, inwieweit Abweichungen im ost- und westdeutschen Scheidungsniveau die Folge einer unterschiedlichen sozialstrukturellen Zusammensetzung der Bevölkerung waren, wird in den empirischen Analysen mit einer Reihe von Kontrollvariablen gearbeitet. Eine dieser Kontrollvariablen ist das Bildungsniveau der Frau. Es wird anhand des höchsten Schulbildungsabschlusses gemessen, den die Befragte zum Zeitpunkt des Interviews erreicht hat. Dabei wird davon ausgegangen, dass die Eheschließung erst nach dem Erreichen dieses Bildungsabschlusses erfolgte. Diese Vorgehensweise ist gerechtfertigt, da lediglich ca. 0,8 Prozent der befragten ost- und westdeutschen Frauen angaben, bereits vor dem Beenden der Schulbildung geheiratet zu haben. Die 
Variable Bildungsabschluss ist unterteilt in a) keiner bzw. niedrig (Haupt/Volksschulabschluss bzw. Polytechnische Oberschule 8. Klasse), b) mittel (Realschulabschluss bzw. Polytechnische Oberschule 10. Klasse) und c) hoch (Fachhochschulreife bzw. allgemeine oder fachgebundene Hochschulreife).

Eine weitere Kontrollvariable ist die, anhand der Kirchgangshäufigkeit gemessene, religiöse Bindung der Frau. Unterschieden wird dabei zwischen: a) mindestens einmal pro Woche, b) mindestens einmal pro Monat, c) selten (nur an kirchlichen Feiertagen bzw. einmal pro Jahr) und d) (fast) nie.

Auch die Beeinflussung des Scheidungsrisikos durch eheliche Instabilität in der Elterngeneration wird in die Analysen einbezogen. Dabei wird eine Scheidung der Eltern jedoch nur berücksichtigt, wenn sie vor dem 18. Geburtstag der Befragten erfolgte.

Weitere zeitunabhängige Kontrollvariablen sind der Familienstand des Partners zum Zeitpunkt der Eheschließung, wobei unterschieden wird zwischen a) ledig bzw. verwitwet und b) geschieden, das Zusammenleben der Partner in Form einer vorehelichen Lebensgemeinschaft, das Heiratsalter und das Heiratsjahr.

Zusätzlich zu diesen zeitunabhängigen Kontrollvariablen geht in die Analysen mit dem Alter des jüngsten Kindes eine zeitabhängig konstruierte Kontrollvariable ein. ${ }^{7}$ Das Alter des jüngsten Kindes wird über sechs Kategorien erfasst: a) kinderlos, b) schwanger, c) 0 bis 3 Jahre, d) 4 bis 7 Jahre, e) 8 Jahre und älter sowie f) Stief-, Pflege- oder Adoptivkinder. ${ }^{8}$

Für alle Variablen, für die es Fälle ohne oder mit fehlerhaften Angaben gibt, wurde eine zusätzliche Kategorie „keine Angabe“ gebildet. Diese geht ebenfalls in die empirischen Analysen ein, sie wird jedoch bei der Darstellung und Interpretation der Ergebnisse nicht berücksichtigt.

\footnotetext{
${ }^{7}$ Bei der Durchführung der Analysen wurden ebenfalls Modelle geschätzt, die anstelle der Variable Alter des jüngsten Kindes die Variable Parität enthielten. Es hat sich jedoch herausgestellt, dass die Parität der Frau weder in Ost- noch in Westdeutschland einen signifikanten Einfluss auf das Scheidungsrisiko hatte. Darüber hinaus ist bei der Untersuchung des Zusammenhangs von Frauenerwerbstätigkeit und Ehestabilität das Alter des jüngsten Kindes von größerer Relevanz.

${ }^{8}$ Für Frauen mit Stief-, Pflege- oder Adoptivkindern wurde eine separate Kategorie gebildet, da in diesen Fällen das Alter des jüngsten Kindes nicht berechnet werden konnte. Der deutsche FFS enthält zwar das Geburtsdatum dieser Kinder, er liefert jedoch keine Informationen darüber ab welchem Zeitpunkt sie im Haushalt der befragten Frau gelebt haben. Darüber hinaus ist anzunehmen, dass es im Hinblick auf den Zusammenhang von Kindern und Scheidungsrisiko Unterschiede gibt zwischen Stief-, Pflege- bzw. Adoptivkindern und leiblichen Kindern.
} 
Der Einfluss der Erwerbsbeteiligung der Frau auf die Stabilität ost- und westdeutscher Ehen wird in dieser Studie mit Hilfe der Ereignisdatenanalyse untersucht. Durch dieses Verfahren lassen sich Übergänge zwischen Zuständen und Verweildauern in Zuständen in einem oder mehreren Bereichen individueller Lebensläufe analysieren. In der Ehestabilitätsforschung wird die Ereignisdatenanalyse seit Beginn der 80er Jahre angewendet und gehört seitdem zu den statistischen Standardverfahren (Wagner 1997: 147; zur Einführung in die Ereignisdatenanalyse vgl. z.B. Blossfeld und Rohwer 2002). Bei dieser Analyse ehelicher Stabilität beginnt die Risikozeit mit der Eheschließung. Sie endet bei Eintritt des beobachteten Ereignisses (Auseinanderziehen der Ehepartner) oder im Falle von Zensierung (Tod eines Ehepartners, unfreiwilliges Auseinanderziehen, deutsche Wiedervereinigung), je nachdem, was zuerst eintritt. Die Risikozeit besteht also aus der Anzahl der Monate, die von der Eheschließung bis zum Eintritt des Ereignisses oder der Zensierung vergehen. Die Scheidungsintensität $\mu(\mathrm{t})$ von Frauen wird in einem multiplikativen, ereignisanalytischen Modell geschätzt. Sie gibt an, mit welcher Wahrscheinlichkeit eine Frau im nächsten Monat eine Scheidung erfahren wird. Dabei wird unterstellt, dass das Risiko einer Ehescheidung über bestimmte Zeitintervalle konstant ist. Der Zeitfaktor ist demnach kategorial. Des Weiteren wird angenommen, dass $\mu(\mathrm{t})$ durch verschiedene zeitunabhängige und zeitabhängige Faktoren beeinflusst wird, die ebenfalls kategorial sind. Das den Analysen zugrunde liegende Modell kann demnach folgendermaßen geschrieben werden:

$$
\begin{aligned}
& \mu(t)_{\text {mnopqrsuvwyz }}=a_{m(t)} b_{n} c_{o} d_{p} e_{q} f_{r} g_{s} h_{u} i_{v} j_{w(t)} k_{y(t)} \\
& \mu(t)_{\text {mopqrsuvwyz }}=a_{m(t)} c_{o} d_{p} e_{q} f_{r} g_{s} h_{u} i_{v} j_{w(t)} k_{y(t)}
\end{aligned}
$$

Während die erste Formel die gemeinsame Analyse des Scheidungsrisikos ost- und westdeutscher Frauen in einem Modell beschreibt und aus diesem Grund einen zusätzlichen Faktor (,Region“ mit den Ausprägungen „Ostdeutschland“ und „Westdeutschland“) enthält, stellt die zweite Formel die Grundlage für die Untersuchung beider Länder in getrennten Modellen dar. Der Faktor $a$ repräsentiert den Effekt der Zeitvariable (Ehedauer in Monaten), $m(t)$ bezeichnet die Intervalle, in denen die Zeitvariable als konstant angenommen wird (0-12, 13-48, 49-84, 85-120, 121-180, 181+ Monate). Die Faktoren $b$ bis i repräsentieren die zeitunabhängigen 
Einflussfaktoren: Region $\left(b_{n}\right)$, Bildungsabschluss $\left(c_{o}\right)$, Scheidung der Eltern $\left(d_{p}\right)$, Kirchgangshäufigkeit $\left(\mathrm{e}_{\mathrm{q}}\right)$, Familienstand des Partners $\left(\mathrm{f}_{\mathrm{r}}\right)$, voreheliche Lebensgemeinschaft $\left(g_{s}\right)$, Heiratsalter $\left(h_{u}\right)$ und Heiratsjahr $\left(i_{v}\right)$. Die Faktoren $j$ und $k$ repräsentieren die zeitabhängigen Einflussfaktoren: Erwerbsbeteiligung $\left(\mathrm{j}_{\mathrm{w}(\mathrm{t})}\right)$ und Alter des jüngsten Kindes $\mathrm{k}_{\mathrm{y}(\mathrm{t})}$ ). Die Aufbereitung des Datensatzes, die Konstruktion der Variablen und die empirischen Analysen wurden mit STATA durchgeführt.

\section{Empirische Analysen}

Das Verfahren der Ereignisdatenanalyse wird im Rahmen dieser Untersuchung in zwei Schritten angewendet. In einem ersten Schritt wird eine vergleichende Analyse des Scheidungsrisikos ost- und westdeutscher Frauen durchgeführt. Dabei soll überprüft werden, inwieweit sich Abweichungen im Scheidungsniveau über die unterschiedliche sozialstrukturelle Zusammensetzung der beiden deutschen Bevölkerungen aufklären lassen und welche Rolle in diesem Zusammenhang die höhere Erwerbsbeteiligung ostdeutscher Frauen spielt. In einem zweiten Schritt wird untersucht, ob es hinsichtlich des Einflusses der Frauenerwerbstätigkeit auf die Ehestabilität länderspezifische Unterschiede gegeben hat.

1. Vergleichende Analyse des Scheidungsrisikos ost- und westdeutscher Frauen

Um zu überprüfen, inwieweit Kompositionseffekte zu Abweichungen im ost- und westdeutschen Scheidungsniveau geführt haben, wird für beide Länder eine gemeinsame Ereignisdatenanalyse durchgeführt. In einem ersten Modell wird über die Variable Region das relative Scheidungsrisiko ost- und westdeutscher Frauen erhoben. Anschließend wird dieses Modell schrittweise um die in Abschnitt IV.2 vorgestellten Kontrollvariablen und die Erwerbsbeteiligungsvariable erweitert. Die Ergebnisse sind in Tabelle 1 dargestellt. 
Tabelle 1: Ereignisanalytisches Modell - relatives Scheidungsrisiko ost- und westdeutscher Frauen $(N=3.153)$

\begin{tabular}{|c|c|c|c|c|c|c|}
\hline & Modell 1 & Modell 2 & Modell 3 & Modell 4 & Modell 5 & Modell 6 \\
\hline \multicolumn{7}{|l|}{ Ehedauer $^{\star}$ ) } \\
\hline 1 Jahr & 0.76 & 0.64 & 0.70 & 0.64 & 0.98 & 0.90 \\
\hline 2 - 4 Jahre & 1.81 & 1.54 & 1.67 & 1.54 & 2.38 & 2.22 \\
\hline 5 - 7 Jahre & 1.76 & 1.51 & 1.63 & 1.52 & 2.38 & 1.96 \\
\hline 8 - 10 Jahre & 1.62 & 1.38 & 1.49 & 1.41 & 2.23 & 1.74 \\
\hline 11 - 15 Jahre & 1.49 & 1.27 & 1.35 & 1.29 & 2.05 & 1.67 \\
\hline über 15 Jahre & 1.69 & 1.44 & 1.52 & 1.49 & 2.42 & 1.90 \\
\hline \multicolumn{7}{|l|}{ Region } \\
\hline Westdeutschland & 1 & 1 & 1 & 1 & 1 & 1 \\
\hline Ostdeutschland & 1.19 * & 1.07 & 1.15 & 1.09 & $0.79 * *$ & $0.81 *$ \\
\hline \multicolumn{7}{|l|}{ Erwerbsbeteiligung } \\
\hline nicht erwerbstätig & & 1 & 1 & 1 & 1 & 1 \\
\hline Teilzeit erwerbstätig & & 1.25 & 1.27 & 1.28 & 1.26 & 1.19 \\
\hline Vollzeit erwerbstätig & & $1.43^{* \star *}$ & $1.45^{\star \star *}$ & $1.43^{* * *}$ & $1.40^{* \star \star}$ & $1.34^{* \star}$ \\
\hline \multicolumn{7}{|l|}{ Bildungsabschluss } \\
\hline keiner bzw. niedrig & & & 1.19 & 1.19 & 1.15 & 1.17 \\
\hline mittel & & & 1 & 1 & 1 & 1 \\
\hline hoch & & & 0.90 & 0.93 & 0.90 & 0.95 \\
\hline \multicolumn{7}{|l|}{ Scheidung der Eltern } \\
\hline nein/nach Alter 18 & & & & 1 & 1 & 1 \\
\hline ja & & & & $2.08^{* * *}$ & $1.95^{* * *}$ & $1.83^{* * *}$ \\
\hline weiß nicht/trifft nicht zu & & & & 1.58 * & $1.55 *$ & 1.48 \\
\hline \multicolumn{7}{|l|}{ Kirchgangshäufigkeit } \\
\hline mind. einmal/Woche & & & & & $0.35 * * \star$ & $0.38 * * *$ \\
\hline mind. einmal/Monat & & & & & $0.47^{* * *}$ & $0.49^{* * *}$ \\
\hline selten & & & & & $0.53^{* * *}$ & $0.57^{* \star *}$ \\
\hline (fast) nie & & & & & 1 & 1 \\
\hline \multicolumn{7}{|l|}{ Familienstand des Partners } \\
\hline ledig/verwitwet & & & & & & 1 \\
\hline geschieden & & & & & & 1.26 \\
\hline \multicolumn{7}{|c|}{ Voreheliche Lebensgemeinschaft } \\
\hline nein & & & & & & 1 \\
\hline ja & & & & & & 1.12 \\
\hline \multicolumn{7}{|l|}{ Heiratsalter } \\
\hline $16-19$ & & & & & & 1 \\
\hline $20-23$ & & & & & & 0.79 ** \\
\hline $24-27$ & & & & & & 0.78 \\
\hline 28 und älter & & & & & & $0.41^{* *}$ \\
\hline \multicolumn{7}{|l|}{ Heiratsjahr } \\
\hline vor 1978 & & & & & & 1 \\
\hline 1978-1981 & & & & & & $1.45^{* * *}$ \\
\hline 1982-1985 & & & & & & $1.73^{* * *}$ \\
\hline 1986-1990 & & & & & & $1.56^{* *}$ \\
\hline \multicolumn{7}{|l|}{ Alter des jüngsten Kindes } \\
\hline kinderlos & & & & & & 1 \\
\hline schwanger & & & & & & $0.50^{* * *}$ \\
\hline 0-3 Jahre & & & & & & $0.76^{* \star}$ \\
\hline 4-7 Jahre & & & & & & $1.38^{* *}$ \\
\hline 8 Jahre und älter & & & & & & 1.38 * \\
\hline Stief-/Pflege-/Adoptivkinder & & & & & & 1.92 ** \\
\hline \multicolumn{7}{|l|}{ Modelgüte } \\
\hline log likelihood & -1793 & -1788 & -1783 & -1764 & -1739 & -1704 \\
\hline $\begin{array}{l}\text { Anmerkungen: (1) Für alle Variable } \\
\text { Kategorie "keine } \\
\left.\text { (2) }{ }^{\star}\right) \text { absolute Risik } \\
\text { (3) }{ }^{\star \star *} p \leq 0,01\end{array}$ & $\begin{array}{l}\text { für die es Fälle } \\
\text { Angabe" eingeg } \\
\text { pro } 1000 \text { Pers } \\
1<p \leq 0,05 \text { * }\end{array}$ & $\begin{array}{l}\text { nne bzw. mit fehle } \\
\text { gen. } \\
\text { enmonate } \\
55<p \leq 0,1\end{array}$ & rhaften Angab & t, ist in die $\mathrm{Ar}$ & e eine zusätzl & \\
\hline
\end{tabular}

Quelle: nationaler FFS für Ost- und Westdeutschland, eigene Berechnungen 
Vernachlässigt man die sozialstrukturelle Zusammensetzung der beiden deutschen Bevölkerungen (Modell 1), so hatten ostdeutsche Frauen ein 19 Prozent höheres Risiko, die Scheidung ihrer ersten Ehe zu erfahren. Westdeutsche Ehen waren im Vergleich zu ostdeutschen Ehen demnach signifikant stabiler.

Durch die Berücksichtigung der unterschiedlich hohen Erwerbsbeteiligung ost- und westdeutscher Frauen (Modell 2) verringern sich vorhandene Abweichungen im Scheidungsrisiko jedoch deutlich. Eine signifikant höhere Stabilität westdeutscher Ehen lässt sich nun nicht mehr feststellen. Dieses Ergebnis zeigt, dass die niedrigere Stabilität ostdeutscher Ehen in entscheidendem Ausmaß durch die höhere Erwerbsbeteiligung ostdeutscher Frauen verursacht wurde.

Die Kontrolle des Einflusses, den der Bildungsabschluss der Frau auf die Ehestabilität hatte (Modell 3), führt dagegen zu einem erneuten Anstieg des relativen Scheidungsrisikos ostdeutscher Frauen. Ursache hierfür ist der positive Zusammenhang zwischen dem Bildungsabschluss der Frau und der Stabilität ehelicher Beziehungen. Aufgrund des höheren Anteils westdeutscher Frauen mit keinem oder niedrigen Bildungsabschluss, erhöht sich das relative Scheidungsrisiko ostdeutscher Frauen durch die Berücksichtigung von Bildungseffekten von 1,07 auf $1,15 .^{9}$

In Modell 4 wird zusätzlich für den Effekt einer Scheidung der Eltern kontrolliert. Da Frauen mit geschiedenen Eltern ein höheres Scheidungsrisiko aufweisen und ein höherer Anteil der ostdeutschen Frauen von einer Scheidung in der Elterngeneration betroffen war, reduziert sich das relative Scheidungsrisiko ostdeutscher Frauen auf 1,09 .

Die ungleiche Verteilung religiöser Bindungen in den beiden deutschen Staaten wird in Modell 5 berücksichtigt. Es zeigt sich, dass die über die Kirchgangshäufigkeit gemessene niedrigere Religiosität ostdeutscher Frauen ein maßgeblicher Grund für die geringere Ehestabilität in Ostdeutschland war. Während das Scheidungsrisiko ostdeutscher Frauen im vierten Modell noch über dem westdeutscher Frauen liegt, sinkt es durch die Berücksichtigung des Einflusses der Kirchgangshäufigkeit deutlich unter das westdeutsche Niveau.

\footnotetext{
${ }^{9}$ Die Verteilung der befragten ost- und westdeutschen Frauen auf die verschiedenen Ausprägungen der Kontrollvariablen und der Erwerbsbeteiligungsvariable ist im Anhang in Tabelle A1 und Tabelle A2 dargestellt.
} 
Die unterschiedliche sozialstrukturelle Zusammensetzung der ost- und westdeutschen Bevölkerung im Hinblick auf den Familienstand des Partners, eine voreheliche Lebensgemeinschaft, das Heiratsalter, das Heiratsjahr und das Alter des jüngsten Kindes (Model 6) hatte dagegen keinen Einfluss auf das relative Scheidungsrisiko beider Staaten.

Insgesamt ist festzuhalten, dass Unterschiede im ost- und westdeutschen Scheidungsniveau in erheblichem Ausmaß die Folge von Kompositionseffekten waren. Während das relative Scheidungsrisiko ostdeutscher Frauen im ersten Modell 19 Prozent über dem westdeutscher Frauen liegt, führt die Berücksichtigung der sozialstrukturellen Zusammensetzung beider Bevölkerungen im sechsten Modell zu einem 19 Prozent niedrigeren Scheidungsrisiko.

2. Der Einfluss der Frauenerwerbstätigkeit auf die Ehestabilität

Um zu untersuchen, welchen Einfluss die Erwerbstätigkeit der Frau auf die Stabilität ost- und westdeutscher Ehen hatte, werden für beide Teile Deutschlands getrennte Modelle geschätzt (siehe Tabelle 2). Erwartungsgemäß zeigt sich sowohl für Ost- als auch für Westdeutschland ein mit der Erwerbsbeteiligung der Frau ansteigendes Scheidungsrisiko. In beiden Ländern wiesen vollzeitbeschäftigte und teilzeitbeschäftigte Frauen gegenüber nicht erwerbstätigen Frauen eine niedrigere Ehestabilität auf. Während der Effekt einer Vollzeiterwerbstätigkeit signifikant ist, trifft dies auf den Effekt einer Teilzeiterwerbstätigkeit nicht zu. 
Tabelle 2: Ereignisanalytisches Modell - relatives Scheidungsrisiko ostdeutscher $(N=1.821)$ und westdeutscher $(N=1.332)$ Frauen

\begin{tabular}{|c|c|c|}
\hline & Ostdeutschland & Westdeutschland \\
\hline \multicolumn{3}{|l|}{ Ehedauer*) } \\
\hline $1 \mathrm{Jahr}$ & 0.60 & 0.73 \\
\hline 2 - 4 Jahre & 1.33 & 2.13 \\
\hline 5 - 7 Jahre & 1.30 & 1.66 \\
\hline 8 - 10 Jahre & 1.31 & 1.14 \\
\hline 11 - 15 Jahre & 0.90 & 1.78 \\
\hline über 15 Jahre & 1.75 & 1.03 \\
\hline \multicolumn{3}{|l|}{ Erwerbsbeteiligung } \\
\hline nicht erwerbstätig & 1 & 1 \\
\hline Teilzeit erwerbstätig & 1.09 & 1.29 \\
\hline Vollzeit erwerbstätig & 1.35 * & $1.43^{* *}$ \\
\hline \multicolumn{3}{|l|}{ Bildungsabschluss } \\
\hline keiner bzw. niedrig & $1.37^{\star \star}$ & 0.94 \\
\hline mittel & 1 & 1 \\
\hline hoch & 1.06 & 0.72 \\
\hline \multicolumn{3}{|l|}{ Scheidung der Eltern } \\
\hline nein/nach Alter 18 & 1 & 1 \\
\hline ja & $1.74^{* * *}$ & $2.06^{* \star *}$ \\
\hline weiß nicht/trifft nicht zu & 1.50 & 1.44 \\
\hline \multicolumn{3}{|l|}{ Kirchgangshäufigkeit } \\
\hline mind. einmal/Woche & 0.62 & $0.26 * * *$ \\
\hline mind. einmal/Monat & 0.96 & $0.30^{* * *}$ \\
\hline selten & 0.83 & $0.37^{\star * \star}$ \\
\hline (fast) nie & 1 & 1 \\
\hline \multicolumn{3}{|l|}{ Familienstand des Partners } \\
\hline ledig/verwitwet & 1 & 1 \\
\hline geschieden & 1.47 * & 0.95 \\
\hline \multicolumn{3}{|l|}{ Voreheliche Lebensgemeinschaft } \\
\hline nein & 1 & 1 \\
\hline ja & 1.18 & 1.04 \\
\hline \multicolumn{3}{|l|}{ Heiratsalter } \\
\hline $16-19$ & 1 & 1 \\
\hline $20-23$ & 0.91 & $0.62 * * *$ \\
\hline $24-27$ & 0.82 & 0.66 * \\
\hline 28 und älter & 0.73 & $0.23^{* *}$ \\
\hline \multicolumn{3}{|l|}{ Heiratsjahr } \\
\hline vor 1978 & 1 & 1 \\
\hline 1978-1981 & $1.40 * *$ & $1.49 * *$ \\
\hline $1982-1985$ & $1.71^{\star \star *}$ & $1.86^{* * *}$ \\
\hline $1986-1990$ & 1.33 & 1.98 ** \\
\hline \multicolumn{3}{|l|}{ Alter des jüngsten Kindes } \\
\hline kinderlos & 1 & 1 \\
\hline schwanger & $0.52^{* *}$ & 0.49 * \\
\hline 0-3 Jahre & 0.82 & 0.70 * \\
\hline 4-7 Jahre & 1.45 * & 1.21 \\
\hline 8 Jahre und älter & 1.05 & $2.13^{\star * \star}$ \\
\hline Stief-/Pflege-/Adoptivkinder & $2.38^{* *}$ & 1.55 \\
\hline \multicolumn{3}{|l|}{ Modelgüte } \\
\hline log likelihood & -1027 & -646 \\
\hline \multicolumn{3}{|c|}{$\begin{array}{l}\text { Anmerkungen: (1) Für alle Variablen, für die es Fälle ohne bzw. mit fehlerhaften Angaben gibt, ist in die Analyse eine zusätzliche } \\
\text { Kategorie "keine Angabe" eingegangen. } \\
\text { (2) *) absolute Risiken pro } 1000 \text { Personenmonate }\end{array}$} \\
\hline
\end{tabular}

Quelle: nationaler FFS für Ost- und Westdeutschland, eigene Berechnungen 
Im Hinblick auf die Kontrollvariablen ist hervorzuheben, dass insbesondere eine Scheidung in der Elterngeneration in beiden Teilen Deutschlands mit einem signifikant höheren Scheidungsrisiko verbunden war. Die Eheschließung mit einem bereits geschiedenen Mann hat dagegen nur in Ostdeutschland zu einer signifikant niedrigeren Ehestabilität geführt. In beiden Ländern ist eine hohe Kirchgangshäufigkeit und ein hohes Alter bei der ersten Eheschließung mit einem niedrigeren Scheidungsrisiko einhergegangen, diese Effekte waren in der BRD jedoch wesentlich stärker und auch nur dort signifikant. Darüber hinaus wiesen sowohl in Ost- als auch in Westdeutschland spätere Eheschließungsjahrgänge eine deutlich niedrigere Ehestabilität auf als frühere (in der DDR traf dies allerdings nur auf Eheschließungsjahrgänge vor 1986 zu). Der positive Einfluss eigener Kinder auf die Stabilität ehelicher Beziehungen war in beiden deutschen Staaten stark abhängig vom Alter des jüngsten Kindes. Lediglich ost- und westdeutsche Mütter, deren jüngstes Kind weniger als vier Jahre alt war, wiesen im Vergleich zu kinderlosen Frauen ein niedrigeres Scheidungsrisiko auf.

Das Schätzen getrennter Modelle für beide Teile Deutschlands erlaubt zwar Aussagen darüber, welchen Einfluss die Erwerbstätigkeit der Frau auf die Stabilität ost- und westdeutscher Ehen hatte, anhand dieser Vorgehensweise kann jedoch nicht überprüft werden, inwieweit es hinsichtlich des Zusammenhangs von Frauenerwerbstätigkeit und Scheidungsrisiko länderspezifische Unterschiede gab. Zur Beantwortung dieser Frage wird, aufbauend auf der für beide Länder gemeinsam durchgeführten Ereignisdatenanalyse (Tabelle 1, Modell 6), eine Interaktion zwischen den Variablen Region und Erwerbsbeteiligung genutzt. Die Ergebnisse sind in Tabelle 3 zusammengefasst.

Tabelle 3: Ereignisanalytisches Modell - relatives Scheidungsrisiko ost- und westdeutscher Frauen (N=3.153), Interaktion zwischen Region und Erwerbsbeteiligung

\begin{tabular}{|c|c|c|}
\hline \multirow{2}{*}{$\begin{array}{l}\text { Erwerbsbeteiligung } \\
\text { der Frau }\end{array}$} & \multicolumn{2}{|c|}{ Region } \\
\hline & Ostdeutschland & Westdeutschland \\
\hline nicht erwerbstätig & 0,84 & 1 \\
\hline Teilzeit erwerbstätig & 0,90 & 1,33 \\
\hline Vollzeit erwerbstätig & 1,07 & $1,47 * *$ \\
\hline
\end{tabular}

Quelle: nationaler FFS für Ost- und Westdeutschland, eigene Berechnungen 
Die Interaktion zwischen den Variablen Erwerbsbeteiligung und Region bestätigt für beide Teile Deutschlands den negativen Zusammenhang von Frauenerwerbstätigkeit und Ehestabilität. Darüber hinaus ist zu erkennen, dass dieser Zusammenhang in Westdeutschland wesentlich stärker war als in Ostdeutschland. Im Vergleich zu nicht erwerbstätigen Frauen hatten Teilzeit erwerbstätige Frauen in Ostdeutschland ein 7 Prozent $(0,90 / 0,84)$, in Westdeutschland dagegen ein 33 Prozent höheres Scheidungsrisiko. Eine Vollzeitbeschäftigung reduzierte die Stabilität ostdeutscher Ehen um 27 Prozent $(1,07 / 0,84)$, die westdeutscher Ehen um 47 Prozent.

Festzuhalten ist, dass es in beiden deutschen Staaten einen negativen Effekt weiblicher Erwerbsbeteiligung auf die Ehestabilität gegeben hat. Während eine Teilzeitbeschäftigung das Scheidungsrisiko ostdeutscher Frauen nur geringfügig erhöhte, hatte diese Erwerbsgruppe in Westdeutschland im Vergleich zu nicht Erwerbstätigen bereits ein deutlich höheres Scheidungsrisiko (auch wenn dieser Effekt nicht signifikant ist). Eine Vollzeitbeschäftigung führte in beiden Ländern zu einem deutlich höheren Scheidungsrisiko, wobei dieser Effekt in Westdeutschland aber stärker war als in Ostdeutschland.

\section{Schlussfolgerungen}

Ziel dieser Studie war es, die Stabilität ost- und westdeutscher Ehen vor der Wiedervereinigung zu vergleichen. Dabei sollte zum einen überprüft werden, auf welche Faktoren Abweichungen im ost- und westdeutschen Scheidungsniveau zurückzuführen sind. Zum anderen wurde untersucht, inwieweit es zwischen den beiden deutschen Staaten hinsichtlich des Einflusses der Frauenerwerbstätigkeit auf die Ehestabilität Unterschiede gegeben hat.

Die vergleichende Analyse des Scheidungsrisikos ost- und westdeutscher Frauen hat gezeigt, dass die Stabilität ehelicher Beziehungen in Ostdeutschland signifikant niedriger war. Verursacht wurde dies zum einen durch die geringere Verbreitung religiöser Bindungen sowie den höheren Anteil ostdeutscher Frauen, die bereits eine Scheidung in der Elterngeneration erlebt hatten, zum anderen durch die höhere Erwerbsbeteiligung ostdeutscher Frauen. Der im Vergleich zu Westdeutschland geringe Anteil von ostdeutschen Frauen mit keinem bzw. mit einem niedrigen Bildungsabschluss hat noch größeren Unterschieden im Scheidungsniveau 
entgegengewirkt. Diese Ergebnisse bestätigen die Vermutung, dass Abweichungen im ost- und westdeutschen Scheidungsniveau unter anderem auf die unterschiedliche sozialstrukturelle Zusammensetzung beider Bevölkerungen zurückzuführen sind. Entgegen der Annahme reduzierten sich vorhandene Ost-West-Unterschiede durch die Berücksichtigung von Kompositionseffekten jedoch nicht, sondern drehten sich um. An die Stelle einer signifikant niedrigeren Stabilität ostdeutscher Ehen trat unter Kontrolle der sozialstrukturellen Zusammensetzung eine signifikant höhere Ehestabilität.

Im Rahmen der - für beide Länder getrennt durchgeführten - Untersuchung des Zusammenhangs von Frauenerwerbstätigkeit und Ehestabilität sollte überprüft werden, ob der negative Effekt einer weiblichen Erwerbsbeteiligung schwächer ist, wenn es sich um eine auf egalitären Rollenvorstellungen beruhende Partnerschaft handelt. Da in der DDR, zumindest in Bezug auf die Erwerbsbeteiligung der Frau, egalitäre Rollenerwartungen vorherrschten, während die gesellschaftlichen und institutionellen Rahmenbedingungen der BRD auf die Förderung des traditionellen Modells eines männlichen Hauptverdieners ausgerichtet waren, wurde angenommen, dass egalitäre Rollenvorstellungen unter ostdeutschen Paaren stärker verbreitet waren als unter westdeutschen Paaren. Auf der Grundlage dieser Annahme wurde vermutet, dass eine Erwerbsbeteiligung der Frau zwar in beiden Ländern mit einem höheren Scheidungsrisiko einhergegangen ist, dass dieser Zusammenhang in der BRD jedoch stärker ausgeprägt war als in der DDR. Die empirischen Analysen haben sowohl für Ost- als auch für Westdeutschland die Annahme eines negativen Einflusses der Frauenerwerbstätigkeit auf die Ehestabilität bestätigt. Darüber hinaus konnte festgestellt werden, dass dieser Zusammenhang für ostdeutsche Frauen schwächer war als für westdeutsche Frauen. Die Ergebnisse dieser Untersuchung unterstützen somit die Vermutung, dass der negative Effekt einer weiblichen Erwerbsbeteiligung auf die Ehestabilität abgeschwächt wird, wenn es sich um eine auf egalitären Rollenvorstellungen beruhende Partnerschaft handelt.

Für das vereinte Deutschland lässt sich auf Grundlage dieser Ergebnisse schlussfolgern, dass weder eine anhaltend hohe Frauenerwerbsbeteiligung (Ostdeutschland) noch eine Zunahme der Frauenerwerbsquote (Westdeutschland) unweigerlich $\mathrm{zu}$ einem Anstieg des deutschen Scheidungsniveaus führen muss. Vorraussetzung hierfür ist jedoch die gesellschaftliche und institutionelle Förderung egalitärer Rollenvorstellungen und damit einhergehend ein Ende der Unterstützung 
traditioneller Rollenerwartungen durch die finanzielle Begünstigung traditioneller Rollenmuster. 


\section{Literatur}

Bast, Kerstin, und Ilona Ostner, 1992: Ehe und Familie in der Sozialpolitik der DDR und BRD - ein Vergleich. S. 228-270 in: W. Schmähl (Hg.): Sozialpolitik im Prozeß der deutschen Vereinigung. Frankfurt am Main: Campus-Verlag.

Becker, Gary S., 1993: A treatise on the family. Enlarged Edition. Cambridge: Harvard University Press.

Becker, Gary S., Elisabeth M. Landes und Robert T. Michael, 1977: An economic analysis of marital instability, The Journal of Political Economy, 85 (6): 1141-1188.

Berghahn, Sabine, und Andrea Fritzsche, 1991: Frauenrecht in Ost- und Westdeutschland: Bilanz, Ausblick. Berlin: Basisdruck-Verlag.

Blossfeld, Hans-Peter, und Götz Rohwer, 2002: Techniques of event history modeling. New approaches to causal analysis. Mahwah, New Jersey: Lawrence Erlbaum Associates.

Booth, Alan, David R. Johnson, Lynn White und John N. Edwards, 1984: Women, outside employment, and marital instability, The american Journal of Sociology, 90 (3): 567-583.

Bothfeld, Silke, Ute Klammer, Christina Klenner, Simone Leiber, Anke Thiel und Astrid Ziegler, 2005: WSI - FrauenDatenReport. Handbuch zur wirtschaftlichen und sozialen Situation von Frauen. Berlin: Edition Sigma.

Brüderl, Josef, und Henriette Engelhardt, 1997: Trennung oder Scheidung? Einige methodologische Überlegungen zur Definition von Eheauflösungen, Soziale Welt, 48: 277-290.

Bundesinstitut für Bevölkerungsforschung, 2003: Das "Family and Fertility Survey" Projekt des BiB. URL: http://www.bib-demographie.de/projekte/ffs/index.html (abgerufen am 28.04.2005).

D'Amico, Ronald, 1983: Status maintenance or status competition? Wife's relative wages as a determinant of labor supply and marital instability, Social Forces, 61 (4): 1186-1205.

Deutsches Institut für Wirtschaftsforschung, 1987: Immer mehr Frauen im Beruf. Zur längerfrisigen Entwicklung des Erwerbsverhaltens von Frauen., Wochenbericht, 54 (29): 393-402.

Dobritz, Jürgen, 1990: Familienstandstafeln für die DDR - Eheschließungen Lediger und Ehescheidungen in der Perioden- und Kohortenmessung. Wiesbaden: Bundesinstitut für Bevölkerungsforschung.

Eurostat (Hg.), 2004: New Cronos Datenbank.

Familiengesetzbuch sowie angrenzende Gesetze und Bestimmungen, 1989, Berlin: 
Staatsverlag der DDR.

Greenstein, Theodore N., 1995: Gender ideology, marital disruption, and the employment of married women, Journal of Marriage and the Family, 57 (1): 31-42.

Greenstein, Theodore N., 1990: Marital disruption and the employment of married women, Journal of Marriage and the Family, 52 (3): 657-676.

Grundgesetz der Bundesrepublik Deutschland, 2005, München: Beck-Texte im dtv.

Hammes, Winfried, 1994: Ehescheidungen 1993, Wirtschaft und Statistik, 12: 978984.

Hartmann, Josef, und Nikolaus Beck, 1999: Berufstätigkeit der Frau und Ehescheidung. S. 179-201 in: Thomas Klein und Johannes Kopp (Hg.): Scheidungsursachen aus soziologischer Sicht. Würzburg: Ergon Verlag.

Heckert, Alex D., Thomas C. Nowak und Kay A. Snyder, 1998: The impact of husbands' and wives' relative earnings on marital disruption, Journal of Marriage and the Family, 60 (3): 690-703.

Hiedemann, Bridget, Olga Suhomlinova und Angela M. O'Rand, 1998: Economic independence, economic status, and emty nest in midlife marriage disruption, Journal of Marriage and the Family, 60 (1): 219-231.

Hill, Paul B., und Johannes Kopp, 1994: Frauenerwerbstätigkeit und Ehescheidung. S. 398-421 in: Petra Beckmann und Gerhard H. Engelbrech (Hg.): Arbeitsmarkt für Frauen 2000 - Ein Schritt vor oder ein Schritt zurück? Kompendium zur Erwerbstätigkeit von Frauen. Nürnberg:

Houle René, 2005: The effects of social-cultural and labor market conditions on marital separation during the early democratic period in Spain. Artikel wurde präsentiert beim Treffen der "Population Association of America 2005" (Philadelphia). URL: http://paa2005.princeton.edu/download.aspx?submissionId= 50268 (abgerufen am 05.04.2005).

Hughes, Diane, Ellen Galinsky und Anne Morris, 1992: The effects of job characteristics on marital quality: specifying linking mechanisms, Journal of Marriage and the Family, 54 (1): 31-42.

Höhn, Charlotte, 1977: Gerichtliche Ehelösungen 1975, Wirtschaft und Statistik, 2: 85-87.

Höhn, Charlotte, 1980: Rechtliche und demographische Einflüsse auf die Entwicklung der Ehescheidungen seit 1946, Zeitschrift für Bevölkerungswissenschaft, 6: 335-371.

Kalmijn, Matthijs, Paul M. De Graaf und Anne-Rigt Poortman, 2004: Interactions between cultural and economic determinants of divorce in The Netherlands, Journal of Marriage and Family, 66 (1): 75-89. 
Kalmijn, Matthijs, Anneke Loeve und Dorien Manting, 2005: Income dynamics in couples and the dissolution of marriage and cohabitation. Artikel wurde präsentiert am Max-Planck-Institut für demografische Forschung, Rostock.

Koch, Petra, und Hans G. Knöbel, 1988: Familienpolitik der DDR im Spannungsfeld zwischen Familie und Berufstätigkeit von Frauen. Pfaffenweiler: CentaurusVerlagsgesellschaft.

Kopp, Johannes, 1994: Scheidung in der Bundesrepublik: Zur Erklärung des langfristigen Anstiegs der Scheidungsraten. Wiesbaden: Westdeutscher Verlag.

Lauterbach, Wolfgang, 1991: Erwerbsmuster von Frauen. Entwicklungen und Veränderungen seit Beginn dieses Jahrhunderts. S. 23-56 in: Karl U. Mayer, Jutta Allmendinger und Johannes Huinink (Hg.): Vom Regen in die Traufe: Frauen zwischen Beruf und Familie. Frankfurt a.M.: Campus Verlag.

Liu, Guiping, und Andres Vikat, 2004: Does divorce risk depend on spouses' relative income? A register-based study of first marriages in Sweden in 1981-1998. MPIDR Working Paper, WP 2004-010, Rostock, Max-Planck-Institut für demografische Forschung.

Mott, Frank L., und Sylvia F. Moore, 1979: The causes of marital disruption among young american women: An interdisciplinary perspective, Journal of Marriage and the Family, 41 (2): 355-365.

Münch, Ursula, 1990: Familienpolitik in der Bundesrepublik Deutschland Maßnahmen, Defizite, Organisation familienpolitischer Staatstätigkeit. Freiburg im Breisgau: Lambertus.

Ono, Hiromi, 1998: Husbands' and Wives' resources and marital dissolution, Journal of Marriage and the Family, 60 (3): 674-689.

Oppenheimer, Valerie K., 1997: Women's employment and the gain to marriage: The specialization and trading model, Annual Review of Sociology, 23: 431-453.

Ott, Notburga, 1992: Verlaufsanalysen zum Ehescheidungsrisiko. S. 227-253 in: Reinhard Hujer, Hilmar Schneider und Wolfgang Zapf (Hg.): Herausforderungen an den Wohlfahrtsstaat im strukturellen Wandel. Frankfurt/New York: Campus Verlag.

Parsons, Talcott, 1971: The social structure of the family. S. 241-274 in: Ruth N. Anshen (Hg.): The family: Its function and destiny. New York: Harper.

Pohl, Katharina, 1995: Familienbildung und Kinderwunsch in Deutschland: Design und Struktur des deutschen FFS. Wiesbaden: Bundesinstitut für Bevölkerungsforschung (BiB).

Rogers, Stacy J., 2004: Dollars, dependency, and divorce: Four perspectives on the role of wives' income, Journal of Marriage and Family, 66 (1): 59-74. 
Ross, Heather L., und Isabel V. Sawhill, 1975: Time of transition. The growth of families headed by women. Washington, D.C.: The Urban Institute.

Sayer, Liana C., und Suzane M. Bianchi, 2000: Women's economic independence and the probability of divorce, Journal of Family Issues, 21 (7): 906-943.

Schäfgen, Katrin, 1998: Die Verdopplung der Ungleichheit. Sozialstruktur und Geschlechterverhältnisse in der Bundesrepublik und in der DDR. URL: http://dochost.rz.hu-berlin.de/dissertation/phil/schaefgen-katrin/PDF/Schaefgen.pdf (abgerufen am 17.01.2005).

South, Scott J., 2001: Time-dependent effects of wives' employment on marital dissolution, American Sociological Review, 66 (2): 226-245.

South, Scott J., und Kim M. Lloyd, 1995: Spousal alternatives and marital dissolution, American Sociological Review, 60 (1): 21-35.

South, Scott J., und Glenna Spitze, 1986: Determinants of divorce over the marital life course, American Sociological Review, 51 (4): 583-590.

Speigner, Wulfram, 1989: Die geburtenfördernde Bevölkerungspolitik der DDR in den 80er Jahren, Wirtschaftswissenschaft, 37 (1): 19-35.

Spitze, Glenna, 1988: Women's employment and family relations: A review, Journal of Marriage and the Family, 50 (3): 595-618.

Staatliche Zentralverwaltung für Statistik (Hg.), 1964-1989: Statistisches Jahrbuch der DDR (1964 - 1989). Berlin: Staatsverlag der Deutschen Demokratischen Republik.

Statistisches Amt der DDR (Hg.), 1990b: Statistisches Jahrbuch der DDR '90. Berlin: Rudolf Haufe Verlag.

Statistisches Bundesamt, 2005: Datenabfrage beim Statistischen Bundesamt, Gruppe VIB.

Statistisches Bundesamt (Hg.), 1989: Statistisches Jahrbuch (1989, 1990, 1992, 1993) für die Bundesrepublik Deutschland. Stuttgart: Metzler-Poeschel.

Trappe, Heike, 1995: Emanzipation oder Zwang? Frauen in der DDR zwischen Beruf, Familie und Sozialpolitik. Berlin: Akademie Verlag.

Wagner, Michael, 1997: Scheidung in Ost- und Westdeutschland. Zum Verhältnis von Ehestabilität und Sozialstruktur seit den 30er Jahren. Frankfurt: Campus Verlag.

Winkler, Gunnar, 1990: Frauenreport '90. Berlin: Verlag Die Wirtschaft. 


\section{Anhang}

Tabelle A1: Verteilung der befragten Frauen auf die verschiedenen Ausprägungen der zeitunabhängigen Variablen (absolute Anzahl und prozentualer Anteil)

\begin{tabular}{|c|c|c|c|c|}
\hline \multirow{2}{*}{$\begin{array}{c}\text { zeitunabhängige } \\
\text { Variablen }\end{array}$} & \multicolumn{2}{|c|}{ Ostdeutschland } & \multicolumn{2}{|c|}{ Westdeutschland } \\
\hline & absolut & prozentual & absolut & prozentual \\
\hline $\begin{array}{l}\text { Bildungsabschluss } \\
\text { keine Angabe } \\
\text { keiner bzw. niedrig } \\
\text { mittel } \\
\text { hoch }\end{array}$ & $\begin{array}{r}14 \\
218 \\
1.316 \\
273\end{array}$ & $\begin{array}{r}0,8 \\
12,0 \\
72,2 \\
15,0\end{array}$ & $\begin{array}{r}14 \\
642 \\
467 \\
209\end{array}$ & $\begin{array}{r}1,0 \\
48,2 \\
35,1 \\
15,7\end{array}$ \\
\hline $\begin{array}{l}\text { Scheidung der Eltern } \\
\text { keine Angabe } \\
\text { nein/nach Alter } 18 \\
\text { ja } \\
\text { weiß nicht/trifft nicht zu }\end{array}$ & $\begin{array}{r}23 \\
1.494 \\
259 \\
45\end{array}$ & $\begin{array}{r}1,3 \\
82,0 \\
14,2 \\
2,5\end{array}$ & $\begin{array}{r}17 \\
1.189 \\
105 \\
21\end{array}$ & $\begin{array}{r}1,3 \\
89,2 \\
7,9 \\
1,6\end{array}$ \\
\hline $\begin{array}{l}\text { Kirchgangshäufigkeit } \\
\text { keine Angabe } \\
\text { mind. einmal/Woche } \\
\text { mind. einmal/Monat } \\
\text { selten } \\
\text { (fast) nie }\end{array}$ & $\begin{array}{r}37 \\
68 \\
67 \\
281 \\
1.368\end{array}$ & $\begin{array}{r}2,0 \\
3,7 \\
3,7 \\
15,5 \\
75,1\end{array}$ & $\begin{array}{r}25 \\
147 \\
209 \\
498 \\
453\end{array}$ & $\begin{array}{r}1,9 \\
11,0 \\
15,7 \\
37,4 \\
34,0\end{array}$ \\
\hline $\begin{array}{l}\text { Familienstand des Partners } \\
\text { keine Angabe } \\
\text { ledig/verwitwet } \\
\text { geschieden }\end{array}$ & $\begin{array}{r}142 \\
1.568 \\
111\end{array}$ & $\begin{array}{r}7,8 \\
86,1 \\
6,1\end{array}$ & $\begin{array}{r}97 \\
1.188 \\
47 \\
\ldots+\ldots\end{array}$ & $\begin{array}{r}7,3 \\
89,2 \\
3,5\end{array}$ \\
\hline $\begin{array}{l}\text { Voreheliche Lebensgemeinschaft } \\
\text { keine Angabe } \\
\text { nein } \\
\text { ja }\end{array}$ & $\begin{array}{r}13 \\
1.238 \\
570\end{array}$ & $\begin{array}{r}0,7 \\
68,0 \\
31,3\end{array}$ & $\begin{array}{r}17 \\
724 \\
591\end{array}$ & $\begin{array}{r}1,3 \\
54,3 \\
44,4\end{array}$ \\
\hline $\begin{array}{l}\text { Heiratsalter } \\
16-19 \\
20-23 \\
24-27 \\
28 \text { und älter }\end{array}$ & $\begin{array}{r}562 \\
1.000 \\
207 \\
52\end{array}$ & $\begin{array}{r}30,8 \\
54,9 \\
11,4 \\
2,9\end{array}$ & $\begin{array}{r}340 \\
603 \\
302 \\
87\end{array}$ & $\begin{array}{r}25,5 \\
45,3 \\
22,7 \\
6,5\end{array}$ \\
\hline $\begin{array}{l}\text { Heiratsjahr } \\
\text { vor } 1978 \\
1978-1981 \\
1982-1985 \\
1986-1990 \\
\end{array}$ & $\begin{array}{l}488 \\
427 \\
446 \\
460 \\
\end{array}$ & $\begin{array}{l}26,8 \\
23,4 \\
24,5 \\
25,3 \\
\end{array}$ & $\begin{array}{l}374 \\
286 \\
345 \\
327 \\
\end{array}$ & $\begin{array}{l}28,1 \\
21,5 \\
25,9 \\
24,5 \\
\end{array}$ \\
\hline Anzahl erster Ehen & 1.821 & 100,0 & 1.332 & 100,0 \\
\hline
\end{tabular}

Quelle: nationaler FFS für Ost- und Westdeutschland 
Tabelle A2: Verteilung der Risikozeit auf die verschiedenen Ausprägungen der zeitabhängigen Variablen (absolute Anzahl und prozentualer Anteil)

\begin{tabular}{|c|c|c|c|c|}
\hline \multirow{2}{*}{ zeitabhängige Variablen } & \multicolumn{2}{|c|}{ Ostdeutschland } & \multicolumn{2}{|c|}{ Westdeutschland } \\
\hline & absolut & prozentual & absolut & prozentua \\
\hline $\begin{array}{l}\text { Erwerbsbeteiligung } \\
\text { keine Angabe } \\
\text { nicht erwerbstätig } \\
\text { Teilzeit erwerbstätig } \\
\text { Vollzeit erwerbstätig }\end{array}$ & $\begin{array}{l}42.162 \\
29.279 \\
13.254 \\
88.059\end{array}$ & $\begin{array}{r}24,4 \\
16,9 \\
7,7 \\
51,0\end{array}$ & $\begin{array}{l}19.670 \\
62.161 \\
13.310 \\
34.414\end{array}$ & $\begin{array}{l}15,2 \\
48,0 \\
10,3 \\
26,6\end{array}$ \\
\hline $\begin{array}{l}\text { Alter des jüngsten Kindes } \\
\text { keine Angabe } \\
\text { kinderlos } \\
\text { schwanger } \\
0-3 \text { Jahre } \\
4-7 \text { Jahre } \\
8 \text { Jahre und älter } \\
\text { Stief-/Pflege-/Adoptivkinder } \\
\end{array}$ & $\begin{array}{r}818 \\
23.134 \\
13.713 \\
66.605 \\
33.942 \\
32.339 \\
2.203 \\
\end{array}$ & $\begin{array}{r}0,5 \\
13,4 \\
7,9 \\
38,6 \\
19,6 \\
18,7 \\
1,3 \\
\end{array}$ & $\begin{array}{r}778 \\
37.777 \\
9.965 \\
40.968 \\
17.767 \\
19.807 \\
2.493 \\
\end{array}$ & $\begin{array}{r}0,6 \\
29,2 \\
7,7 \\
31,6 \\
13,7 \\
15,3 \\
1,9 \\
\end{array}$ \\
\hline $\begin{array}{l}\text { Anzahl beobachteter } \\
\text { Personenmonate }\end{array}$ & 172.754 & 100,0 & 129.555 & 100,0 \\
\hline
\end{tabular}

Quelle: nationaler FFS für Ost- und Westdeutschland 